\title{
Intraday variability in compact extragalactic radio sources
}

\section{VLA observations}

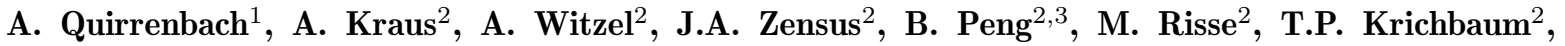 \\ R. Wegner ${ }^{2}$, and C.E. Naundorf ${ }^{2}$ \\ 1 University of California San Diego, Dept. of Physics, Center for Astrophysics and Space Sciences, Mail Code 0424, La Jolla, \\ CA 92093-0424, U.S.A. \\ 2 Max-Planck-Institut für Radioastronomie, Auf dem Hügel 69, 53121 Bonn, Germany \\ 3 Beijing Astronomical Observatory, Chinese Academy of Science, Beijing 100080, PR China
}

Received July 22; accepted October 26, 1999

\begin{abstract}
We present the data of three observing campaigns with the VLA performed to study intraday variability in compact extragalactic radio sources. The first campaign (May 1989) lasted 5 days; total intensity as well as linear polarization data were obtained at $2,3.6,6$, and $20 \mathrm{~cm}$ wavelength for 9 program sources. In the second observing run (February 1990), a five-antenna subarray of the VLA was used for 25 days to monitor the sources $0716+714,0917+624$, and $0954+658$, with dense sampling at the same four wavelengths. In the third run (October 1992), which lasted 22 days, 8 program sources were monitored at $3.6,6$, and $20 \mathrm{~cm}$ wavelength, and 2 additional sources at $6 \mathrm{~cm}$ only, again with a five-antenna subarray. We present summary tables, light curves, and structure functions from these data sets.
\end{abstract}

Key words: galaxies: active — quasars: general — radio continuum: galaxies

\section{Introduction}

Since its discovery in 1985 (cf. Witzel et al. 1986; Heeschen et al. 1987) it has been shown that intraday variability (IDV, i.e., flux-density variations on time scales less than a day) is a common, but complex phenomenon among compact flat-spectrum extragalactic radio sources. It is detected in a large fraction of this class of sources (e.g. Quirrenbach et al. 1992). Variations with amplitudes of up to a factor of two have been reported in the radio as well as optical bands (Quirrenbach et al. 1992; Wagner \& Witzel 1995; Kedziora-Chudczer et al.

Send offprint requests to: A. Kraus,

e-mail: akraus@mpifr-bonn.mpg.de
1997). The occurrence of IDV appears to be correlated with the milliarcsecond structure of the sources: it is very common in objects dominated by a compact VLBI core, but is not normally found in sources with a prominent VLBI jet (Quirrenbach et al. 1992). In parallel to the variability of the total flux density, variations of the linearly polarized flux density and the polarization angle have been observed in many sources (Quirrenbach et al. 1989b, Kraus et al. 1999a,c; Kraus et al., in preparation). Both correlations (e.g. 0716+714) and anti-correlations (e.g. $0917+624)$ between the total and the polarized flux density have been seen (Wegner 1994). In 0716+714 and $0954+658$ there is evidence for a correlation between the rapid flux density variability in the radio and the optical regime (Quirrenbach et al. 1991; Wagner et al. 1993). Such an effect would severely constrain any models of IDV and rule out propagation effects as their only cause. From light travel time arguments, it is clear that IDV gives insight in very small parts of the AGN (light days to light hours), if it is intrinsic to the sources. In that case it implies apparent brightness temperatures of $10^{18} \mathrm{~K}$ to $10^{21} \mathrm{~K}$, far in excess of the inverse Compton limit of $10^{12} \mathrm{~K}$ (Kellermann \& Pauliny-Toth 1969).

In this paper we present the results of three observing campaigns carried out with the Very Large Array in order to study intraday variations in a number of compact radio sources. We point out that these sources were selected mainly on the basis of noticeable variations in earlier observations with the Effelsberg $100 \mathrm{~m}$ telescope; they do not form a statistically complete sample in any sense. (For an analysis of IDV in statistically complete samples see Quirrenbach et al. 1992.) In the next section the observations and data reduction procedures are described, afterwards the methods of statistical analysis are introduced. The properties of all program sources are 
listed in Sect. 5. All observations are summarized in tabular form, and for all program sources the light curves are plotted. In addition, for the sources which showed IDV in total intensity, we present the structure functions.

Additional observations of intraday variability performed with the Effelsberg $100 \mathrm{~m}$ telescope, and a more detailed interpretation of the available data on intraday radio variability will be presented in forthcoming Papers II and III in this series (Kraus et al., in preparation).

Subsets of the data presented in here have already been published (Quirrenbach et al. 1989b; Qian et al. 1991; Wagner et al. 1996; Kraus et al. 1999b). In addition to the observations of extragalactic sources, two radio stars (UX Ari, V711 Tau) were included in the October 1992 observing run (Elias et al. 1995).

\section{Observations and data reduction}

\subsection{Observations}

The dates of the observations, the array configurations, and the observing frequencies are listed in Table 1. For the first experiment we used the full VLA for about 5 days. The array was divided into two similar sub-arrays; one of them was used for observations at $0.33 \mathrm{GHz}, 1.49$, and $4.86 \mathrm{GHz}$, the other at 8.44 and $15.0 \mathrm{GHz}$. Both other campaigns were made during ongoing $\mathrm{D} \rightarrow \mathrm{A}$ reconfiguration times of the VLA with sub-arrays of five antennae for periods of three to four weeks. Since the information from our observations with a five-antenna subarray was insufficient for polarization calibration ${ }^{1}$, we could obtain reliable polarization information only for the first experiment. The data at $0.33 \mathrm{GHz}$ turned out to be heavily affected by interference and confusion problems, and could not be meaningfully searched for variability.

The observational procedure was as follows: Every source was observed for approximately five minutes per frequency, then we changed to another source. At regular intervals, we included non-variable steep-spectrum sources $(1311+678$ and $1634+628)$ to monitor and correct for any elevation-dependent and time-dependent effects (this procedure had successfully been used before in IDV observations at the $100 \mathrm{~m}$ telescope, cf. Heeschen et al. 1987;

\footnotetext{
${ }^{1}$ During $\mathrm{D} \rightarrow \mathrm{A}$ reconfiguration antennae are moved from the innermost to the outermost stations of the VLA. Our subarray therefore consisted of a mix of very short and very long baselines. In addition, the composition of the subarray changed frequently, as an antenna was checked out to be moved, and replaced by another antenna that had just been repositioned. The positions of the newly checked-in antennae were often not known precisely at the time of our observations, resulting in unusually large phase errors. These problems could be overcome in the analysis of the total intensities, but prevented us from performing a sufficiently reliable polarization calibration.
}

Quirrenbach et al. 1989a). In addition, the steep-spectrum sources are useful to determine the measurement errors, since they do not show any genuine variations on short timescales (Heeschen et al. 1987).

\subsection{Reduction of the total intensity data}

The primary data are $10 \mathrm{~s}$ outputs from the correlator for each antenna pair, separately for the four polarization combinations (RR, LL, RL, and LR), and two adjacent $50 \mathrm{MHz}$ IF bands. For the calibration, as a first step, erroneous $10 \mathrm{~s}$ intervals were eliminated. Hereafter, we have not followed the usual data analysis procedures for VLA observations, since amplitude self-calibration would level out the variations we have been looking for. Since for sources dominated by a strong point-like core - as are most of the sources observed here - the phase is zero by definition, we have performed phase self-calibration for every source first. Subsequently, a (one-day) mean amplitude gain factor has been derived using non-variable sources like $1311+678$, which were linked to an absolute flux density scale (Baars et al. 1977; Ott et al. 1994) by frequent observations of the primary calibrators 3C 286 and $3 \mathrm{C} 48$. The choice of a constant amplitude gain factor for each one-day block of data has the advantage that systematic amplitude changes are not leveled out and can be corrected more precisely later.

During the first few hours of the May 1989 observing run, we observed the field around each source with the full array in B configuration. Maps of the sources were obtained from these data with standard VLA data reduction procedures, including full self-calibration and Högbom cleaning. The clean components corresponding to extended emission or confusing sources (i.e., all clean components but those at the origin of the image plane) were Fourier transformed back into the $u v$ plane, and subtracted from the measured visibilities. (We assume that the flux density in the outer components does not show any significant variations.) This procedure isolates the pointlike cores of the sources. Thus, we have been able to avoid systematic artificial variations due to extended structure without losing any data (as would happen by simply discarding data outside a limited $u v$ range). Extended structure has to be taken into account for $0716+714, \quad 0836+710, \quad 0954+658, \quad 1642+690$, and $1928+738$ at $\lambda=6 \mathrm{~cm}$, and for $0716+714,1642+690$, $1928+738$, and $2007+777$ at $\lambda=20 \mathrm{~cm}$. Corrections for confusing sources within the field-of-view were made for $0917+624$ and $0954+658$.

After this correction and a second pass of editing spurious sections of the data, each five-minute scan was incoherently averaged over time, baselines, polarization (RR and LL), and IF bands. Due to the point-like structure of the sources (after our structure correction where necessary), the mean correlated flux density is equal to the 
Table 1. Summary of the observing campaigns. The number in the last column gives the number of flat-spectrum sources observed; i.e., the steep-spectrum calibrators are not included in these figures

\begin{tabular}{lllc}
\hline date & array configuration & $\nu[\mathrm{GHz}]$ & number of sources \\
\hline May 4, 1989 - May 9, 1989 & full array, B $\rightarrow$ B/C-reconfiguration & $0.33,1.49,4.86,8.44,15.0$ & 9 \\
Jan. 29, 1990 - Feb. 23, 1990 & sub-array, 5 antennae & $1.49,4.86,8.44,15.0$ & 3 \\
Oct. 2, 1992 - Oct. 23, 1992 & sub-array, 5 antennae & $1.49,4.86,8.44$ & $10(+2$ stars $)$ \\
\hline
\end{tabular}

core flux density. The flux density in the extended structure was now added back to the data, to obtain the total flux density of the source (and enable comparison to flux densities measured e.g. at the $100 \mathrm{~m}$ telescope). Eventually, we removed spurious elevation-dependent and time-dependent effects in the light curves, using correction functions derived from observations of the non-variable sources $0836+710,1311+678$, and $1634+628$.

The errors are composed of the statistical errors from the averaging and a contribution from the residual systematic fluctuations. The level of these uncorrected residuals was determined from the apparent variations of the calibrator source 3C 286 and of the steep-spectrum sources. Typical standard deviations were found to be in the range of $0.5 \%$ to $1.0 \%$ (depending on the wavelength) of the mean value, with no significant difference between the individual non-variable sources. Only for the $2 \mathrm{~cm}$ data was the standard deviation significantly higher, about $1.3 \%$ to $2 \%$.

\subsection{Reduction of the polarization data}

For the polarization data (experiment in May 1989) we performed phase and amplitude calibration as described above. The Stokes parameters $Q$ and $U$ were derived from the cross correlation between the LHC and RHC polarizations, with gain factors carried over from the analysis of the total intensity data. The circular polarization of these sources is negligible, cf. Weiler \& de Pater (1983). $Q$ and $U$ were then converted into polarized flux density $P$ and polarization position angle $\chi$. The source $0836+710$ was used to determine the instrumental polarization, assuming that its polarized intensity and polarization angle are constant in time. In this case, the polarization vector $\boldsymbol{P}=(Q, U)$ should describe a circle in the $Q-U$-plane around the origin due to the parallactic rotation. The shift from the origin to the actual center gives the vector of the instrumental polarization which was subtracted from the measured polarization vector for all sources. A Faraday rotation correction was applied to the data at $\lambda=20 \mathrm{~cm}$. Since the global phase difference between LHC and RHC is unknown, all polarization angles $\chi$ may be rotated by a constant value. This was calibrated with the main calibrator $3 \mathrm{C} 286$ for which a polarization position angle of $33^{\circ}$ was assumed at all wavelengths. Eventually, systematic effects were corrected using the non-variable sources.
Table 2. Observations in May 1989, total intensity

\begin{tabular}{|c|c|r|r|r|r|c|}
\hline \multicolumn{2}{|c}{$\lambda=20 \mathrm{~cm}$} & \multicolumn{1}{c}{ TOT } & \multicolumn{1}{c|}{$T=5 \mathrm{~d}$} & $m_{0}=0.55 \%$ \\
\hline \hline Source & $N$ & $I[\mathrm{Jy}]$ & $m[\%]$ & $\chi_{\mathrm{r}}^{2}$ & $Y[\%]$ & type \\
\hline $0716+714$ & 38 & 0.87 & 1.33 & 5.01 & 3.63 & II \\
$0836+710$ & 75 & 3.81 & 0.51 & 0.85 & & 0 \\
$0917+624$ & 101 & 1.13 & 4.27 & 44.26 & 12.70 & II \\
$0954+658$ & 112 & 0.99 & 1.66 & 8.29 & 4.71 & II \\
$1642+690$ & 39 & 1.14 & 1.06 & 2.96 & 2.73 & II \\
$1749+701$ & 38 & 0.78 & 0.62 & 1.16 & & 0 \\
$1803+784$ & 38 & 2.13 & 1.80 & 11.80 & 5.13 & I \\
$1928+738$ & 38 & 3.42 & 0.60 & 1.08 & & 0 \\
$2007+777$ & 36 & 1.19 & 1.13 & 3.87 & 2.95 & II \\
\hline
\end{tabular}

\begin{tabular}{|c|c|r|r|r|r|c|}
\hline \multicolumn{2}{|c|}{$\lambda=6 \mathrm{~cm}$} & \multicolumn{1}{c|}{ TOT } & \multicolumn{1}{c|}{$T=5 \mathrm{~d}$} & \multicolumn{3}{c|}{$m_{0}=0.50 \%$} \\
\hline \hline Source & $N$ & $I[\mathrm{Jy}]$ & $m[\%]$ & \multicolumn{1}{c|}{$\chi_{\mathrm{r}}^{2}$} & $Y[\%]$ & type \\
\hline $0716+714$ & 39 & 0.62 & 3.52 & 54.12 & 10.45 & $\mathrm{I}$ \\
$0836+710$ & 79 & 2.11 & 0.34 & 0.50 & & 0 \\
$0917+624$ & 98 & 1.51 & 4.38 & 85.09 & 13.06 & $\mathrm{II}$ \\
$0954+658$ & 115 & 1.26 & 0.93 & 3.73 & 2.34 & $\mathrm{II}$ \\
$1642+690$ & 39 & 1.20 & 0.94 & 3.68 & 2.38 & $\mathrm{II}$ \\
$1749+701$ & 37 & 0.65 & 1.28 & 7.37 & 3.55 & $\mathrm{II}$ \\
$1803+784$ & 38 & 3.48 & 0.58 & 1.56 & & 0 \\
$1928+738$ & 37 & 3.65 & 0.55 & 1.37 & & 0 \\
$2007+777$ & 39 & 2.16 & 0.81 & 2.95 & 1.92 & $\mathrm{I}$ \\
\hline
\end{tabular}

\begin{tabular}{|c|c|r|r|r|r|c|}
\hline \multicolumn{2}{|c}{$\lambda=3.6 \mathrm{~cm}$} & \multicolumn{1}{c}{ TOT } & \multicolumn{3}{c|}{$T=5 \mathrm{~d}$} & \multicolumn{3}{c|}{$m_{0}=0.70 \%$} \\
\hline \hline Source & $N$ & $I[\mathrm{Jy}]$ & $m[\%]$ & $\chi_{\mathrm{r}}^{2}$ & $Y[\%]$ & type \\
\hline $0716+714$ & 37 & 0.69 & 2.83 & 15.56 & 8.24 & II \\
$0836+710$ & 75 & 1.55 & 0.60 & 0.70 & & 0 \\
$0917+624$ & 96 & 1.57 & 2.76 & 6.91 & 8.00 & $\mathrm{II}$ \\
$0954+658$ & 112 & 1.22 & 1.08 & 2.23 & 2.47 & $\mathrm{II}$ \\
$1642+690$ & 38 & 1.17 & 0.96 & 1.78 & & 0 \\
$1749+701$ & 38 & 0.52 & 2.06 & 1.87 & & 0 \\
$1803+784$ & 37 & 3.68 & 0.63 & 0.74 & & 0 \\
$1928+738$ & 38 & 3.71 & 0.63 & 0.76 & & 0 \\
$2007+777$ & 36 & 2.35 & 0.53 & 0.55 & & 0 \\
\hline
\end{tabular}

\begin{tabular}{|c|c|r|r|c|c|c|}
\hline \multicolumn{2}{|c|}{$\lambda=2 \mathrm{~cm}$} & \multicolumn{1}{c|}{ TOT } & \multicolumn{1}{c|}{$T=5 \mathrm{~d}$} & \multicolumn{3}{c|}{$m_{0}=1.30 \%$} \\
\hline \hline Source & $N$ & $I[\mathrm{Jy}]$ & $m[\%]$ & $\chi_{\mathrm{r}}^{2}$ & $Y[\%]$ & type \\
\hline $0716+714$ & 39 & 0.97 & 2.10 & 2.58 & 4.93 & $\mathrm{II}$ \\
$0836+710$ & 77 & 1.37 & 0.88 & 0.45 & & 0 \\
$0917+624$ & 96 & 1.51 & 2.08 & 2.49 & 4.87 & $\mathrm{II}$ \\
$0954+658$ & 111 & 1.15 & 1.72 & 1.70 & 3.36 & $\mathrm{II}$ \\
$1642+690$ & 38 & 1.13 & 1.38 & 1.09 & & 0 \\
$1749+701$ & 38 & 0.47 & 1.56 & 1.43 & & 0 \\
$1803+784$ & 38 & 3.56 & 1.46 & 1.25 & & 0 \\
$1928+738$ & 37 & 3.48 & 1.51 & 1.32 & & 0 \\
$2007+777$ & 39 & 2.30 & 1.40 & 1.14 & & 0 \\
\hline
\end{tabular}


Table 3. Observations in May 1989; polarized intensity and polarization angle

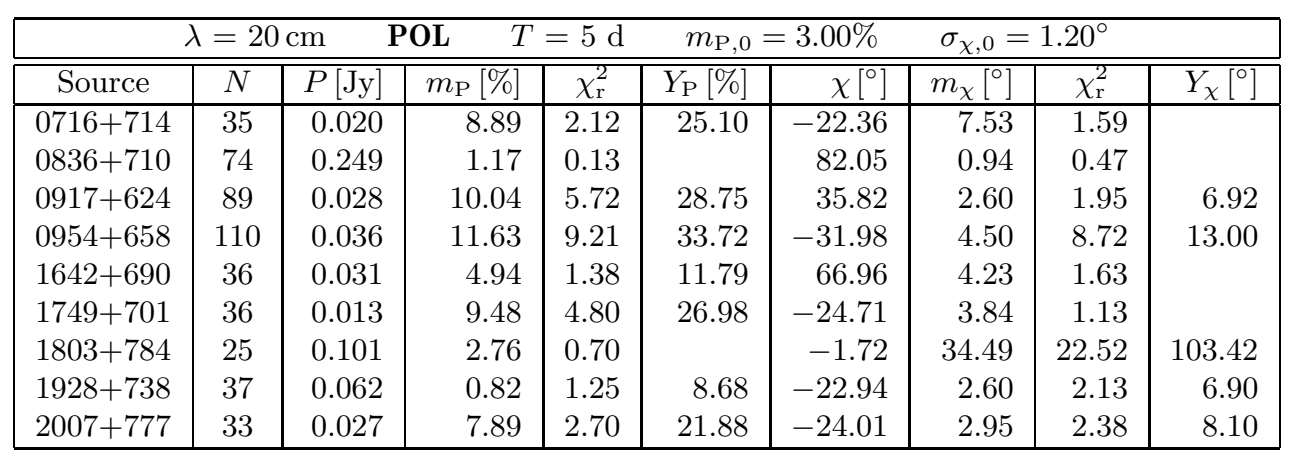

\begin{tabular}{|c|c|c|r|r|r|r|r|r|r|}
\hline \multicolumn{4}{|c|}{$\lambda=6 \mathrm{~cm}$ POL $T=5 \mathrm{~d}$} & \multicolumn{1}{c|}{$m_{\mathrm{P}, 0}=1.50 \%$} & \multicolumn{1}{|c|}{$\sigma_{\chi, 0}=0.80^{\circ}$} \\
\hline \hline Source & $N$ & $P[\mathrm{Jy}]$ & $m_{\mathrm{P}}[\%]$ & \multicolumn{1}{c|}{$\chi_{\mathrm{r}}^{2}$} & $Y_{\mathrm{P}}[\%]$ & $\chi\left[^{\circ}\right]$ & $m_{\chi}\left[^{\circ}\right]$ & $\chi_{\mathrm{r}}^{2}$ & $Y_{\chi}\left[^{\circ}\right]$ \\
\hline $0716+714$ & 39 & 0.031 & 10.92 & 27.38 & 32.45 & -6.15 & 1.77 & 2.44 & 4.74 \\
$0836+710$ & 73 & 0.191 & 1.24 & 0.64 & & 103.55 & 0.67 & 0.69 & \\
$0917+624$ & 100 & 0.016 & 27.51 & 101.2 & 82.40 & -4.39 & 12.00 & 57.64 & 35.91 \\
$0954+658$ & 113 & 0.121 & 2.06 & 1.75 & 4.25 & -20.58 & 0.51 & 0.39 & \\
$1642+690$ & 39 & 0.041 & 8.46 & 17.83 & 24.97 & -50.11 & 1.90 & 3.41 & 5.17 \\
$1749+701$ & 36 & 0.019 & 6.66 & 9.40 & 19.47 & -71.27 & 2.92 & 1.41 & \\
$1803+784$ & 38 & 0.140 & 4.25 & 7.32 & 11.93 & 83.28 & 1.48 & 1.62 & \\
$1928+738$ & 15 & 0.067 & 5.80 & 9.55 & 16.82 & 74.63 & 4.53 & 1.85 & \\
$2007+777$ & 36 & 0.047 & 9.96 & 31.28 & 29.53 & -75.56 & 3.32 & 7.43 & 9.66 \\
\hline
\end{tabular}

\begin{tabular}{|c|c|c|c|c|c|c|c|c|c|}
\hline \multicolumn{3}{|c|}{$\lambda=3.6 \mathrm{~cm}$} & POL & $T=5 \mathrm{~d}$ & \multicolumn{2}{|c|}{$m_{\mathrm{P}, 0}=3.00 \%$} & \multicolumn{3}{|c|}{$\sigma_{\chi, 0}=1.00^{\circ}$} \\
\hline Source & $N$ & $P[\mathrm{Jy}]$ & $m_{\mathrm{P}}[\%]$ & $\bar{\chi}_{\mathrm{r}}^{2}$ & $\overline{Y_{\mathrm{P}}[\%]}$ & $\bar{\chi}\left[^{\circ}\right]$ & $m_{\chi}\left[^{0}\right]$ & $\overline{\chi_{\mathrm{r}}^{2}}$ & $\overline{Y_{\chi}\left[{ }^{\circ}\right]}$ \\
\hline $0716+714$ & 35 & 0.043 & 10.21 & 11.33 & 29.29 & 0.23 & 2.09 & 2.46 & 5.51 \\
\hline $0836+710$ & 71 & 0.143 & 2.38 & 0.61 & & 106.84 & 0.42 & 0.73 & \\
\hline $0917+624$ & 96 & 0.018 & 15.32 & 14.26 & 45.08 & -18.82 & 6.20 & 9.97 & 18.37 \\
\hline $0954+658$ & 107 & 0.136 & 2.33 & 0.58 & & -21.88 & 0.92 & 0.61 & \\
\hline $1642+690$ & 37 & 0.078 & 4.86 & 2.53 & 11.49 & -25.98 & 1.45 & 1.39 & \\
\hline $1749+701$ & 36 & 0.022 & 5.06 & 2.21 & 12.21 & -72.39 & 4.89 & 2.83 & 14.35 \\
\hline $1803+784$ & 22 & 0.146 & 2.90 & 0.85 & & -80.58 & 6.68 & 9.47 & 19.83 \\
\hline 1928 & 0 & & & & & & & & \\
\hline $2007+777$ & 34 & 0.108 & 4.98 & 2.50 & 11.94 & -71.09 & 1.87 & 2.68 & 4.74 \\
\hline
\end{tabular}

\begin{tabular}{|c|c|c|r|c|r|r|r|r|r|r|}
\hline \multicolumn{4}{|c|}{$\lambda=2 \mathrm{~cm}$} & \multicolumn{1}{c|}{ POL } & \multicolumn{2}{c|}{$T=5 \mathrm{~d}$} & $m_{\mathrm{P}, 0}=3.50 \%$ & \multicolumn{3}{c|}{$\sigma_{\chi, 0}=1.00^{\circ}$} \\
\hline \hline Source & $N$ & $P[\mathrm{Jy}]$ & $m_{\mathrm{P}}[\%]$ & $\chi_{\mathrm{r}}^{2}$ & $Y_{\mathrm{P}}[\%]$ & $\chi\left[^{\circ}\right]$ & $m_{\chi}\left[{ }^{\circ}\right]$ & $\chi_{\mathrm{r}}^{2}$ & $Y_{\chi}\left[^{\circ}\right]$ \\
\hline $0716+714$ & 37 & 0.066 & 10.34 & 6.91 & 29.20 & 1.42 & 2.31 & 2.03 & 6.25 \\
$0836+710$ & 72 & 0.083 & 4.87 & 1.30 & & 11.34 & 1.27 & 0.59 & \\
$0917+624$ & 95 & 0.026 & 13.41 & 5.64 & 38.83 & -18.83 & 5.91 & 2.56 & 17.47 \\
$0954+658$ & 106 & 0.130 & 3.91 & 0.95 & & -20.09 & 1.15 & 0.72 & \\
$1642+690$ & 37 & 0.103 & 6.00 & 2.68 & 14.62 & -18.82 & 2.02 & 1.76 & 1.71 \\
$1749+701$ & 0 & & & & & & & & \\
$1803+784$ & 24 & 0.113 & 5.81 & 2.28 & 13.92 & -70.97 & 9.72 & 6.17 & 29.01 \\
$1928+738$ & 32 & 0.128 & 9.35 & 4.73 & 26.00 & 65.40 & 1.68 & 1.37 & \\
$2007+777$ & 36 & 0.163 & 6.19 & 2.36 & 15.32 & -77.10 & 1.42 & 0.74 & \\
\hline
\end{tabular}

The resulting errors were in the range of $3 \%$ to $5 \%$ for the polarized flux density and $3^{\circ}$ to $5^{\circ}$ for the polarization angle. It turned out that - in contrast to the total intensity data - a significant fraction of the polarization data was very noisy. Most of these data points have been discarded, resulting in a sparser sampling for some polarization light curves. For the sources $1749+701$ (at $\lambda=$ $2 \mathrm{~cm}$ ) and $1928+738$ (at $\lambda=3.6 \mathrm{~cm}$ ), we could not get any reliable polarization data. 


\section{Statistical analysis}

For the data analysis we use the modulation index $m$, which is related to the mean flux density $\langle S\rangle$ and the rms flux density variations $\sigma_{S}$ by

$m[\%]=100 \cdot \frac{\sigma_{S}}{<S>}$.

It provides a measure of the strength of the variations observed, although it does not take into account the error of the individual measurement. As a criterion whether a source is variable or not, we perform a $\chi^{2}$ test with

$\chi^{2}=\sum_{i=1}^{N}\left(\frac{S_{i}-<S>}{\Delta S_{i}}\right)^{2}$,

where the $S_{i}$ are the individual flux densities and $\Delta S_{i}$ their errors, e.g. Bevington \& Robinson (1992). Actually, we determine the probability that a constant function is a good fit to the data. Only sources for which the probability that they can be fitted by a constant function is $\leq 0.1 \%$, are considered to be variable.

To compare the modulation indices at different wavelengths and epochs, and to obtain a quantity that is independent of the measurement noise, we define the variability amplitude for the variable sources as

$Y[\%]=3 \sqrt{m^{2}-m_{0}^{2}}$,

where $m_{0}$ is the modulation index of a non-variable source (see Heeschen et al. 1987). The factor 3 is somewhat arbitrary, but makes $Y$ to be of the order of the peak-to-peak amplitude of the variations.

For the polarized intensity the corresponding quantities are

$m_{P}[\%]=100 \cdot \frac{\sigma_{P}}{<P>}$,

$Y_{P}[\%]=3 \sqrt{m_{P}^{2}-m_{P, 0}^{2}}$;

and for the polarization angle we use

$m_{\chi}\left[^{\circ}\right]=\sigma_{\chi}$,

$Y_{\chi}\left[^{\circ}\right]=3 \sqrt{\sigma_{\chi}^{2}-\sigma_{\chi, 0}^{2}}$.

We note that this description of the polarization variability is somewhat problematic because the polarization measurements are more strongly affected by systematic effects than the total intensity data, and because the statistical errors have a Gaussian distribution in the Stokes parameters $Q$ and $U$, not in $P$ and $\chi$. We have nevertheless chosen the above description, since the separation of the variability of $P$ and $\chi$ is more relevant for a physical interpretation of the variations.

Structure functions can be used for the analysis of the characteristics of the variability, and to search for typical timescales and periodicities. The first order structure function $D(\tau)$ is defined as (see Simonetti et al. 1985):

$D(\tau)=<(S(t)-S(t+\tau))^{2}>_{\mathrm{t}}$ with $<$. $>_{t}$ being the mean over time again. Since our data are unevenly sampled, we derived the structure functions using an interpolation algorithm. For any given time lag $\tau$, the value of $S(t+\tau)$ is calculated by linear interpolation of the two adjacent data points. Each structure function was derived twice, firstly starting from the beginning of the time series, and secondly starting at the end and proceeding backwards. This provides a rough assessment of the errors caused by the interpolation process (but not of the systematic uncertainty due to the finite length of the observations).

A typical timescale in the lightcurve (i.e., the time between a minimum and a maximum or vice versa) is indicated by a maximum of the structure function, while a period in the lightcurve causes a minimum of the structure function (e.g. Heidt \& Wagner 1996). Following the nomenclature of Heeschen et al. (1987), structure functions can be used to define variability types, depending on the observed timescale. A source whose structure function reaches a maximum within the observing period is called type II (i.e., a fast-varying source). In the case of a monotonically increasing structure function (i.e., when the source shows no typical timescale less than the total observing period), it is assigned type I. Since this definition is problematic for longer observing campaigns as the ones in February 1990 and October 1992, we define a variable source whose structure function reaches a maximum or plateau within $\tau \leq 2$ days as being of type II (cf. Wagner \& Witzel 1995), otherwise type I. Nonvariable sources are assigned type 0 .

\section{Results}

An overview over the results is given in Tables 2, 4, and 5 , which list the source name, number of observations, the mean value of the flux density, the modulation index, the reduced $\chi^{2}$ value, and the variability amplitude. In the last column of each table the variability type is given. The latter was defined using structure functions, as described above. Similarly, the results for the linear polarization data (May 1989 only) are presented in Table 3. The tables listing total intensities are labeled TOT, those with polarization data POL.

The resulting light curves (of the flat-spectrum sources) are plotted in Figs. 2 to 23. For the May 1989 data the polarized flux density and the polarization angle are also given. As an example for the steep-spectrum sources (which were used to check for any instrumental effects and to correct those), see the source $1311+678$ in Fig. 1 which was taken from the October 1992 observations at $6 \mathrm{~cm}$. Its modulation index was $0.44 \%$.

For all total intensity light curves of the variable sources, structure functions were derived and plotted in Figs. 24 to 40 . 


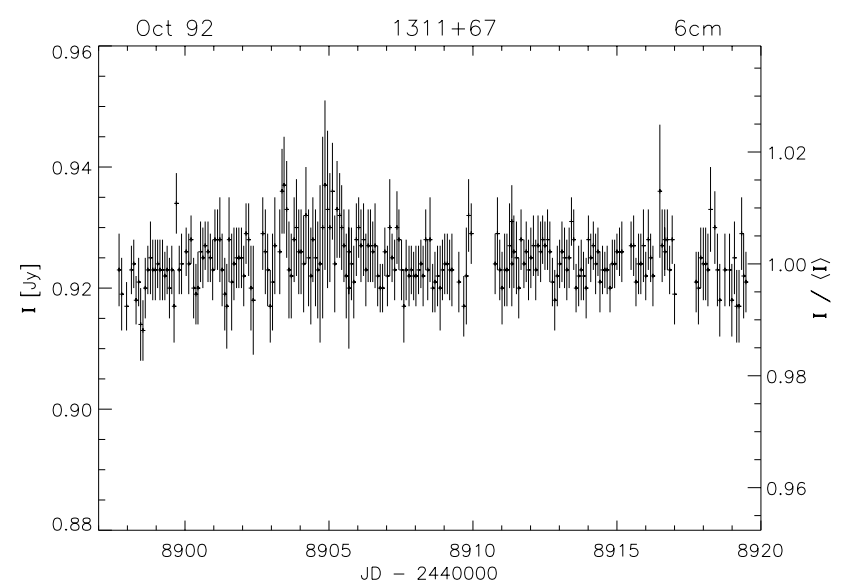

Fig. 1. Steep-spectrum quasar $1311+678$, observed in October 1992 at $6 \mathrm{~cm}$. After elevation-dependent and time-dependent correction a high accuracy was reached

\section{Individual sources}

Here we list briefly specific properties for the individual flat-spectrum sources observed. The optical identifications and redshifts are taken from Kühr et al. (1981) and Stickel et al. (1994).

- 0153+744: This quasar $(z=2.338)$ was observed in October 1992. There are some indications of variability on the $1 \%$-level, but these are not strong enough to pass the $\chi^{2}$ test.

- 0235+164: This BLLac object $(z=0.940)$ was observed in October 1992. Strong short-timescale variations have been found, especially an event which is unusual in that it appears to be faster and stronger at $20 \mathrm{~cm}$ than at shorter wavelengths. Possible models for these peculiar variations have been discussed in detail by Kraus et al. (1999b).

- 0333+321: Observed only at $\lambda=6 \mathrm{~cm}$ in October 1992 . This source (a quasar, $z=1.258$ ) was included as phase calibrator for the radio star UX Ari, and showed IDV on the $3 \%$-level.

- 0336-019: This quasar $(z=0.852)$ was measured only at $\lambda=6 \mathrm{~cm}$ in October 1992, as phase calibrator for V711 Tau. It showed IDV on the 5\%-level.

- 0716+714: Observed in all three campaigns, this source (a BL Lac object with unknown redshift) shows pronounced variations at all frequencies and in linear polarization. There seems to be a correlation between the flux density at various frequencies (except for $\lambda=20 \mathrm{~cm}$ ), and between total and polarized flux density.

- 0804+499: In Effelsberg data obtained in April 1990 this quasar ( $z=1.433)$ showed the highest variability amplitude observed so far in sources of the northern hemisphere (Quirrenbach et al. 1992). Therefore, it was included in the October 1992 VLA campaign,
Table 4. Observations in February 1990, total intensity

\begin{tabular}{|c|c|r|r|c|c|c|}
\hline \multicolumn{2}{|c|}{$\lambda=20 \mathrm{~cm}$} & \multicolumn{1}{c|}{ TOT } & \multicolumn{3}{c|}{$T=25 \mathrm{~d}$} & \multicolumn{3}{c|}{$m_{0}=0.70 \%$} \\
\hline \hline Source & $N$ & $I[\mathrm{Jy}]$ & $m[\%]$ & $\chi_{\mathrm{r}}^{2}$ & $Y[\%]$ & type \\
\hline $0716+714$ & 186 & 0.87 & 3.58 & 14.52 & 10.54 & $\mathrm{I}$ \\
$0917+624$ & 330 & 1.22 & 4.43 & 23.63 & 13.13 & $\mathrm{I}$ \\
$0954+658$ & 373 & 1.03 & 4.19 & 25.49 & 12.40 & $\mathrm{I}$ \\
\hline
\end{tabular}

\begin{tabular}{|c|c|r|r|r|r|c|}
\hline \multicolumn{2}{|c|}{$\lambda=6 \mathrm{~cm}$} & \multicolumn{1}{c|}{ TOT } & \multicolumn{1}{c|}{$T=25 \mathrm{~d}$} & $m_{0}=0.50 \%$ \\
\hline \hline Source & $N$ & $I[\mathrm{Jy}]$ & $m[\%]$ & \multicolumn{1}{c|}{$\chi_{\mathrm{r}}^{2}$} & $Y[\%]$ & type \\
\hline $0716+714$ & 209 & 0.57 & 5.44 & 22.39 & 16.25 & $\mathrm{I} / \mathrm{II}$ \\
$0917+624$ & 378 & 1.46 & 3.66 & 38.19 & 10.88 & $\mathrm{II}$ \\
$0954+658$ & 416 & 1.23 & 1.52 & 7.57 & 4.31 & $\mathrm{I}$ \\
\hline
\end{tabular}

\begin{tabular}{|c|c|r|r|c|r|c|}
\hline \multicolumn{2}{|c}{$\lambda=3.6 \mathrm{~cm}$} & \multicolumn{1}{c}{ TOT } & \multicolumn{1}{c|}{$T=25 \mathrm{~d}$} & \multicolumn{3}{c|}{$m_{0}=1.00 \%$} \\
\hline \hline Source & $N$ & $I[\mathrm{Jy}]$ & $m[\%]$ & $\chi_{\mathrm{r}}^{2}$ & $Y[\%]$ & type \\
\hline $0716+714$ & 186 & 0.54 & 3.96 & 8.79 & 11.48 & I \\
$0917+624$ & 330 & 1.53 & 2.55 & 6.13 & 7.04 & $\mathrm{II}$ \\
$0954+658$ & 359 & 1.19 & 1.78 & 3.13 & 4.41 & $\mathrm{II}$ \\
\hline
\end{tabular}

\begin{tabular}{|c|c|r|r|c|c|c|}
\hline \multicolumn{2}{|c}{$\lambda=2.0 \mathrm{~cm}$} & \multicolumn{1}{c}{ TOT } & \multicolumn{3}{c|}{$T=25 \mathrm{~d}$} & \multicolumn{3}{c|}{$m_{0}=2.00 \%$} \\
\hline \hline Source & $N$ & $I[\mathrm{Jy}]$ & $m[\%]$ & $\chi_{\mathrm{r}}^{2}$ & $Y[\%]$ & type \\
\hline $0716+714$ & 138 & 0.64 & 6.33 & 7.57 & 18.01 & $\mathrm{I} / \mathrm{II}$ \\
$0917+624$ & 268 & 1.53 & 2.19 & 0.95 & & 0 \\
$0954+658$ & 299 & 1.16 & 2.24 & 1.05 & & 0 \\
\hline
\end{tabular}

in which it showed rapid variability, although "only" on a level of a few percent.

- 0836+710: No variations are seen in this quasar $(z=$ 2.172 ) which is not surprising because this source is characterized by a pronounced, long jet (e.g. Otterbein et al. 1998; Lobanov et al. 1998). Therefore it can be (and was) used as secondary calibrator; it is particularly useful as a polarization calibrator because of its high degree of polarization $(\sim 9 \%$ at $6 \mathrm{~cm})$.

- 0917+624: One of the most strongly variable sources (a quasar, $z=1.446$ ) in total flux density and linear polarization $(P$ and $\chi$; e.g. Quirrenbach et al. 1989b; Kraus et al. 1999c). It was observed in May 1989 and February 1990, and showed pronounced IDV which seems to be correlated at wavelengths $\lambda \leq 6 \mathrm{~cm}$. The variations at $\lambda=20 \mathrm{~cm}$ are somewhat slower. The polarized flux density seems to be anti-correlated with the total flux density. Both intrinsic variations (Qian et al. 1991) and propagation effects in the interstellar medium (Rickett et al. 1995) have been explored as potential explanations of IDV in this source.

- 0954+658: A BL Lac object $(z=0.367)$ showing variability on a level of a few percent, somewhat stronger at $\lambda=20 \mathrm{~cm}$ in February 1990. Except at $\lambda=20 \mathrm{~cm}$ it showed no significant variability in the linear polarization.

- 1642+690: This quasar $(z=0.751)$ was observed in May 1989, and showed IDV at $\lambda=20 \mathrm{~cm}$ and $6 \mathrm{~cm}$. 
Table 5. Observations in October 1992, total intensity

\begin{tabular}{|c|c|r|r|r|r|c|}
\hline \multicolumn{2}{|c|}{$\lambda=20 \mathrm{~cm}$} & \multicolumn{1}{c|}{ TOT } & \multicolumn{1}{c|}{$T=22 \mathrm{~d}$} & \multicolumn{3}{c|}{$m_{0}=0.70 \%$} \\
\hline \hline Source & \multicolumn{1}{c|}{$N$} & $I[\mathrm{Jy}]$ & $m[\%]$ & \multicolumn{1}{c|}{$\chi_{\mathrm{r}}^{2}$} & $Y[\%]$ & type \\
\hline $0153+744$ & 102 & 1.81 & 0.74 & 0.40 & & 0 \\
$0235+164$ & 121 & 1.57 & 7.41 & 153.8 & 22.14 & $\mathrm{I}$ \\
$0716+714$ & 229 & 0.75 & 2.51 & 3.87 & 7.23 & $\mathrm{I}$ \\
$0804+499$ & 134 & 1.00 & 3.10 & 14.04 & 9.06 & $\mathrm{I}$ \\
$0836+170$ & 218 & 3.60 & 0.54 & 0.72 & & 0 \\
$1749+701$ & 222 & 0.73 & 1.39 & 4.34 & 3.61 & $\mathrm{I}$ \\
$1803+784$ & 219 & 1.81 & 1.12 & 4.11 & 2.64 & $\mathrm{I}$ \\
$2007+777$ & 218 & 1.12 & 4.44 & 39.69 & 13.16 & $\mathrm{I}$ \\
\hline
\end{tabular}

\begin{tabular}{|c|c|r|r|r|r|c|}
\hline \multicolumn{2}{|c|}{$\lambda=6 \mathrm{~cm}$} & \multicolumn{1}{c|}{ TOT } & \multicolumn{1}{c|}{$T=22 \mathrm{~d}$} & \multicolumn{3}{c|}{$m_{0}=0.70 \%$} \\
\hline \hline Source & \multicolumn{1}{c}{$N$} & $I[\mathrm{Jy}]$ & $m[\%]$ & \multicolumn{1}{c|}{$\chi_{\mathrm{r}}^{2}$} & $Y[\%]$ & type \\
\hline $0153+744$ & 107 & 1.32 & 0.78 & 1.07 & & 0 \\
$0235+164$ & 117 & 4.05 & 4.77 & 77.35 & 14.14 & $\mathrm{I}$ \\
$0333+321$ & 59 & 1.84 & 1.50 & 5.61 & 3.99 & $\mathrm{II}$ \\
$0333-019$ & 58 & 2.49 & 2.45 & 11.56 & 7.05 & $\mathrm{II}$ \\
$0716+714$ & 215 & 0.77 & 5.13 & 58.90 & 15.24 & $\mathrm{II}$ \\
$0804+499$ & 157 & 1.13 & 1.94 & 11.79 & 5.44 & $\mathrm{II}$ \\
$0836+170$ & 229 & 2.13 & 0.49 & 0.56 & & 0 \\
$1749+701$ & 226 & 0.66 & 2.27 & 13.69 & 6.49 & $\mathrm{I}$ \\
$1803+784$ & 227 & 2.06 & 2.80 & 30.06 & 8.15 & $\mathrm{I}$ \\
$2007+777$ & 231 & 2.55 & 2.72 & 21.97 & 7.90 & $\mathrm{I}$ \\
\hline
\end{tabular}

\begin{tabular}{|c|c|r|r|r|r|c|}
\hline \multicolumn{2}{|c|}{$\lambda=3.6 \mathrm{~cm}$} & \multicolumn{1}{c|}{ TOT } & \multicolumn{1}{c|}{$T=22 \mathrm{~d}$} & \multicolumn{3}{c|}{$m_{0}=0.80 \%$} \\
\hline \hline Source & $N$ & $I[\mathrm{Jy}]$ & $m[\%]$ & \multicolumn{1}{c|}{$\chi_{\mathrm{r}}^{2}$} & $Y[\%]$ & type \\
\hline $0153+744$ & 97 & 0.77 & 0.80 & 0.78 & & 0 \\
$0235+164$ & 108 & 5.23 & 2.01 & 6.13 & 5.54 & $\mathrm{II}$ \\
$0716+714$ & 216 & 0.84 & 8.24 & 53.37 & 24.60 & $\mathrm{I}$ \\
$0804+499$ & 139 & 1.16 & 2.08 & 6.57 & 5.77 & $\mathrm{II}$ \\
$0836+170$ & 217 & 1.93 & 0.72 & 0.69 & & 0 \\
$1749+701$ & 204 & 0.64 & 1.34 & 2.04 & 3.21 & $\mathrm{II}$ \\
$1803+784$ & 219 & 2.20 & 2.25 & 7.37 & 6.32 & $\mathrm{II}$ \\
$2007+777$ & 210 & 2.61 & 0.82 & 0.97 & & 0 \\
\hline
\end{tabular}

- 1749+701: One of the first sources in which IDV was discovered (Heeschen et al. 1987). This BL Lac object $(z=0.770)$ showed weak variations in May 1989 and stronger IDV in October 1992.

- 1803+784: This source (classified variously as BL Lac object or quasar, $z=0.684$ ) showed only slow variability (type I) in most observations. At $\lambda=20 \mathrm{~cm}$ strong variability of the polarization angle has been found.

- 1928+738: A quasar $(z=0.302)$ which has a pronounced jet structure similar to $0836+710$. Correspondingly, it did not show any IDV in total intensity. Only in linear polarization weak variability has been seen.

- 2007+777: A BLLac object $(z=0.342)$ showing mostly slow variations.

\section{Discussion}

We have presented data from three observing campaigns carried out at the VLA to investigate intraday variability of compact extragalactic radio sources. In total, we observed 14 flat-spectrum sources (some repeatedly); out of these only 3 (namely $0153+744,0836+710$, and $1928+738$ ) did not show IDV in any of the observations. This confirms that this phenomenon occurs consistently in a significant number of compact radio sources (with a flat spectrum), although clear statistical conclusions are precluded by our target selection, which favored objects that had previously shown fast variability. Also, the detailed properties vary strongly between sources. In most cases, the variations in total flux density are accompanied by similar variability of the linear polarization. Very fast variations (i.e., on timescales $\leq 2$ days, type II) occur in about $40 \%$ of all cases. One should keep in mind, however, that this is a small, by no means complete sample of sources. More detailed statistics, including observations of a larger number of sources with the Effelsberg $100 \mathrm{~m}$ telescope, will be presented separately (Kraus et al., in preparation).

The size of a variable source can be derived from typical timescales assuming an intrinsic origin of the variability. In this case the linear size cannot be much larger than $c \cdot \Delta t$; with the usual synchrotron theory this leads to an expression for the brightness temperature of the source (cf. Wagner \& Witzel 1995)

$T_{\mathrm{B}}[\mathrm{K}]=4.510^{10} \cdot S[\mathrm{Jy}] \cdot\left(\frac{\lambda[\mathrm{cm}] \cdot D_{\mathrm{L}}[\mathrm{Mpc}]}{\Delta t[\mathrm{~d}] \cdot(1+z)}\right)^{2}$.

Therefore, intraday variations imply brightness temperatures of $10^{16}-10^{19} \mathrm{~K}$, or even $10^{21} \mathrm{~K}$ for the very rapid variations in PKS $0405-385$ observed by KedzioraChudczer et al. (1997). This is a severe violation of the inverse Compton limit of $10^{12} \mathrm{~K}$ (Kellermann \& PaulinyToth 1969). We note that for the standard shock-in-jet model the observed (variability) brightness temperatures should be reduced by a factor $\mathcal{D}^{3}$ where $\mathcal{D}$ is the Doppler factor of the source (Blandford 1990). However, to bring down the brightness temperatures to the inverse Compton limit, Doppler factors of order 20-200 are needed. Such high Doppler factors are not supported by other observations (e.g. Witzel et al. 1988; Ghisellini et al. 1993), and they pose severe theoretical problems (e.g. Begelman et al. 1994). Since the discovery of IDV a large number of models have been developed to explain this phenomenon. Intrinsic explanations have used e.g. the motion of a compact structure (shock) in an underlying relativistic jet (Blandford \& Königl 1979; Marscher \& Gear 1985; Qian et al. 1991; Qian et al., in preparation) or the reconnection of magnetic field lines and coherent emission processes (Benford 1992; Lesch \& Pohl 1992). Alternatively, IDV could be caused by extrinsic mechanisms, e.g. scattering in the interstellar medium (ISS, e.g. Rickett et al. 1995). Since the 
strength of ISS depends strongly on the galactic latitude (Rickett 1990), the measurement of a complete sample with a broad distribution in galactic latitude can be used for a crucial test for scattering models. However, none of the models discussed is fully capable of explaining all observed properties of IDV.

In forthcoming papers (Kraus et al., in preparation) we will present the IDV observations carried out at the $100 \mathrm{~m}$ telescope of the MPIfR in Effelsberg and a complete statistical analysis of all available observations.

Acknowledgements. We thank the NRAO staff for their expert assistance and advice, and A. Patnaik and K. Otterbein for help during the data analysis. The VLA is a facility of the NRAO, which is operated by Associated Universities Inc., under cooperative agreement with the NSF.

\section{References}

Baars J.W.M., Genzel R., Pauliny-Toth I.I.K., Witzel A., 1977, A\&A 61, 99

Begelman M.C., Rees M.J., Sikora M., 1994, ApJ 429, L57

Benford G., 1992, ApJ 391, L59

Bevington P.R., Robinson D.K., 1992, Data reduction and error analysis for the physical sciences. McGraw-Hill, New York

Blandford R.D., 1990, in: Blandford R.D., Netzer H., Woltjer L. (eds.), Active Galactic Nuclei, Saas-Fee Advanced Course 20. Springer, p. 161

Blandford R.D., Königl A., 1979, ApJ 232, 34

Elias N.M., Quirrenbach A., Witzel A., et al., 1995, ApJ 439, 983

Ghisellini G., Padovani P., Celotti A., Maraschi L., 1993, ApJ 407, 65

Heeschen D.S., Krichbaum T.P., Schalinski C.J., Witzel A., 1987, AJ 94, 1493

Heidt J., Wagner S.J., 1996, A\&A 305, 42

Kedziora-Chudczer L., Jauncey D.L., Wieringa M.H., Walker M.A., Nicolson G.D., Reynolds J.E., Tzioumis A.K., 1997, ApJ 490, L9

Kellermann K.I., Pauliny-Toth I.I.K., 1969, ApJ 155, L71
Kraus A., Krichbaum T.P., Witzel A., 1999a, in: Takalo L., Sillanpää A. (eds.), BL Lac phenomenon, ASP Conf. Ser. 159, 49

Kraus A., Quirrenbach A., Lobanov A., et al., 1999b, A\&A 344,807

Kraus A., Witzel A., Krichbaum T.P., 1999c, New Astron. Rev. (in press)

Kühr H., Witzel A., Pauliny-Toth I.I.K., Nauber U., 1981, A\&AS 45, 367

Lesch H., Pohl M., 1992, A\&A 254, 29

Lobanov A.P., Krichbaum T.P., Witzel A., et al., 1998, A\&A 340, L60

Marscher A.P., Gear W.K., 1985, ApJ 298, 114

Ott M., Witzel A., Quirrenbach A., et al., 1994, A\&A 284, 331

Otterbein K., Krichbaum T.P., Kraus A., et al., 1998, A\&A 334,489

Qian S.J., Quirrenbach A., Witzel A., et al., 1991, A\&A 241, 15

Quirrenbach A., Witzel A., Krichbaum T.P., et al., 1989a, Nat 337,442

Quirrenbach A., Witzel A., Krichbaum T.P., et al., 1992, A\&A 258,279

Quirrenbach A., Witzel A., Qian S.J., et al., 1989b, A\&A 226, L1

Quirrenbach A., Witzel A., Wagner S.J., et al., 1991, ApJ 372, L71

Rickett B.J., 1990, ARA\&A 28, 561

Rickett B.J., Quirrenbach A., Wegner R., et al., 1995, A\&A 293,479

Simonetti J.H., Cordes J.M., Heeschen D.S., 1985, ApJ 296, 46 Stickel M., Meisenheimer K., Kühr H., 1994, A\&AS 105, 211

Wagner S.J., Witzel A., 1995, ARA\&A 33, 163

Wagner S.J., Witzel A., Heidt J., et al., 1996, AJ 111, 2187

Wagner S.J., Witzel A., Krichbaum T.P., et al., 1993, A\&A 271,344

Wegner R., 1994, Ph.D. Thesis. University of Bonn

Weiler K.W., de Pater I., 1983, ApJS 52, 293

Witzel A., Heeschen D.S., Schalinski C.J., Krichbaum T.P., 1986, Mitt. Astron. Ges. 65, 239

Witzel A., Schalinski C.J., Johnston K.J., Biermann P.L., et al., 1988, A\&A 206, 245 
A. Quirrenbach et al.: Intraday variability in compact extragalactic radio sources. I.
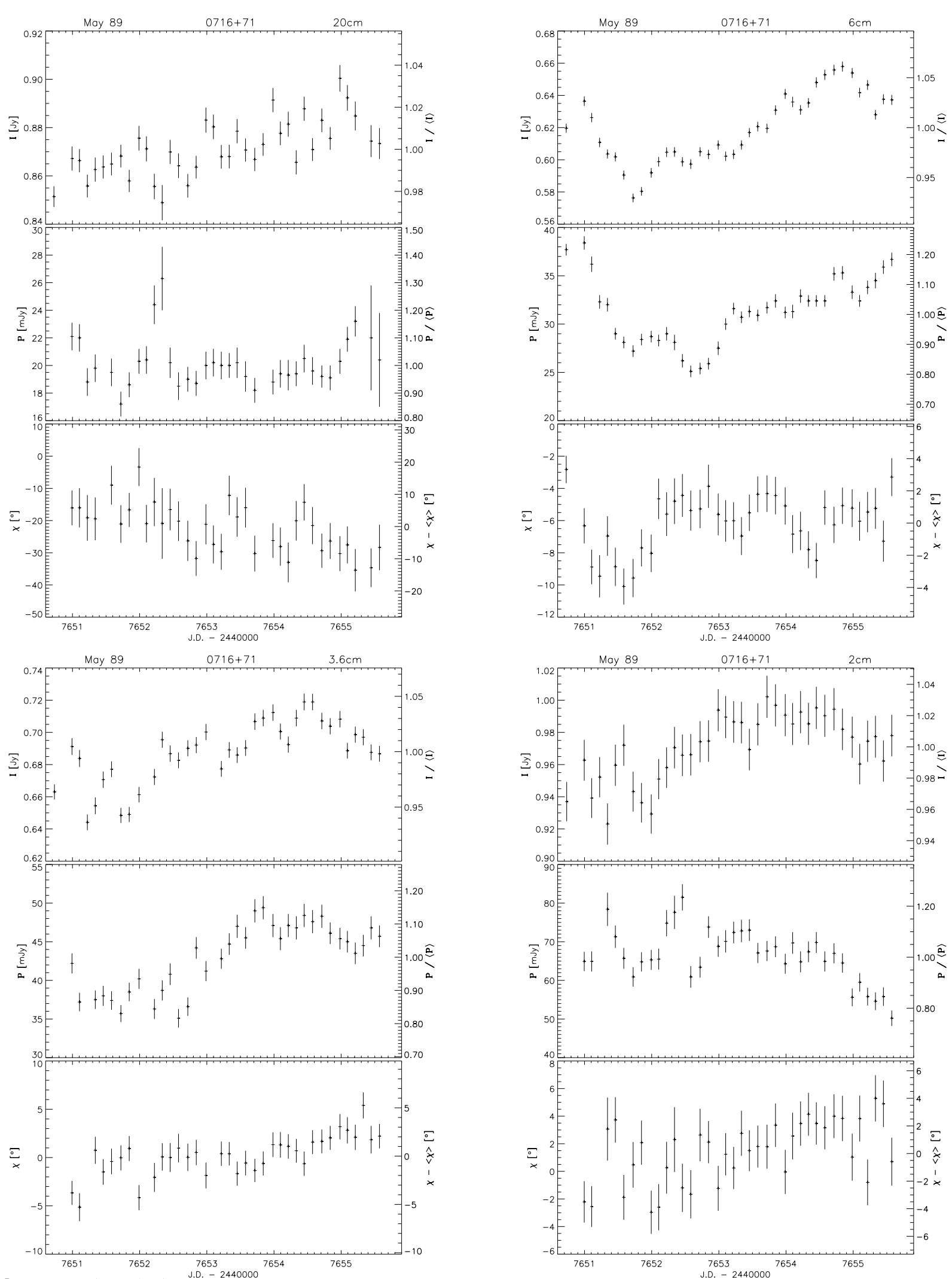

Fig. 2. May 1989; 0716+714 

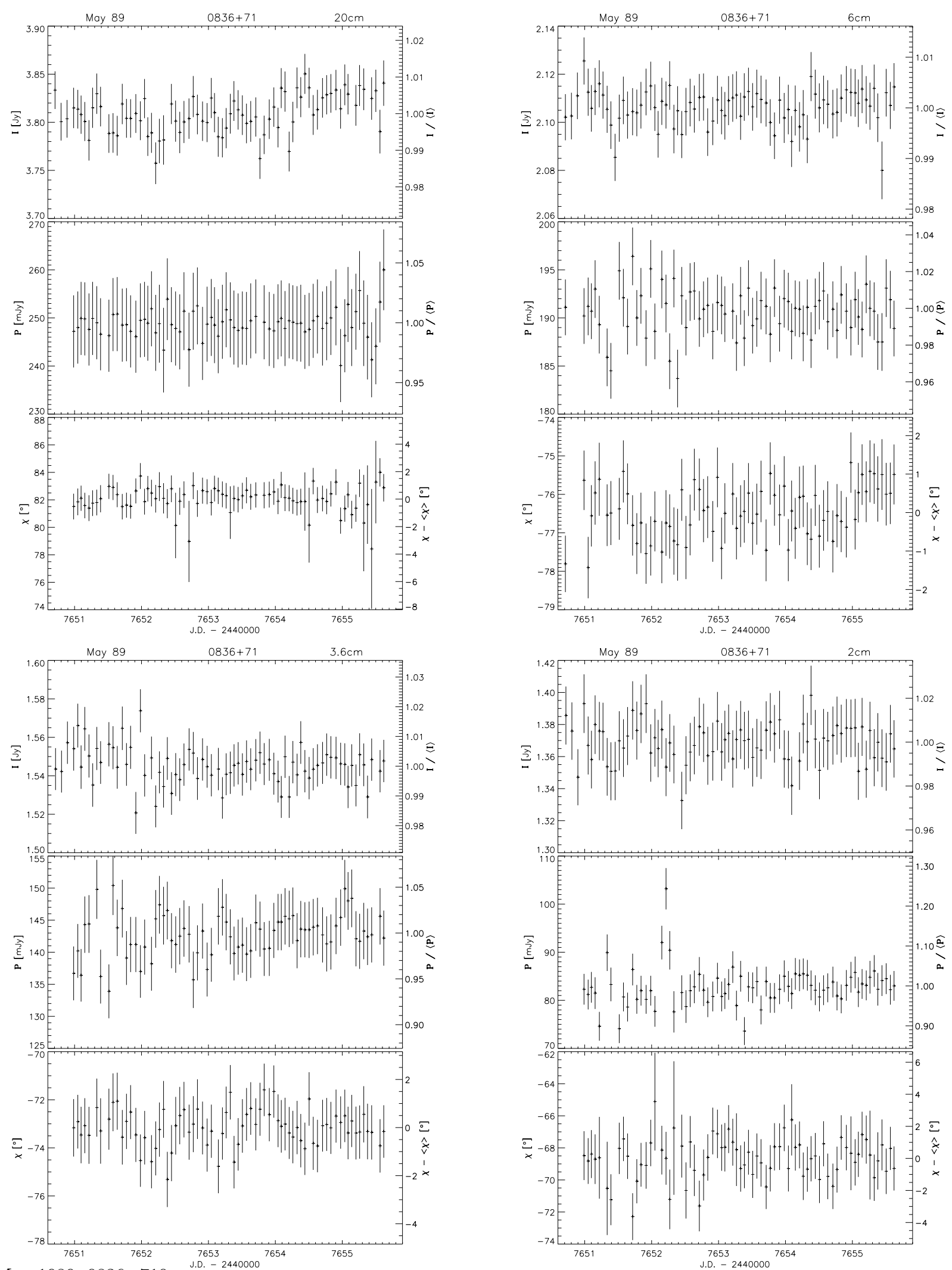

Fig. 3. May 1989; 0836+710 
A. Quirrenbach et al.: Intraday variability in compact extragalactic radio sources. I.
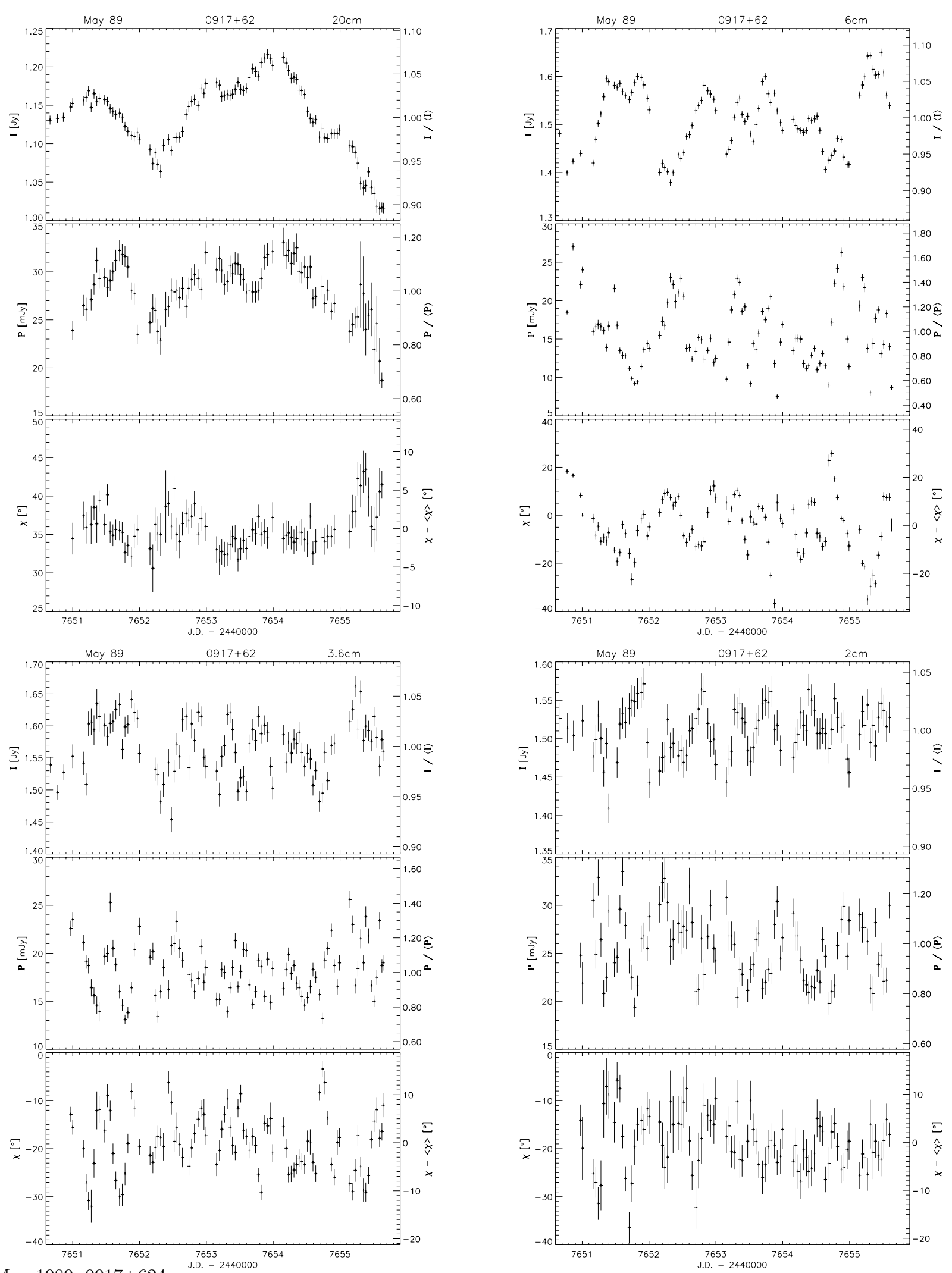

Fig. 4. May 1989; 0917+624 

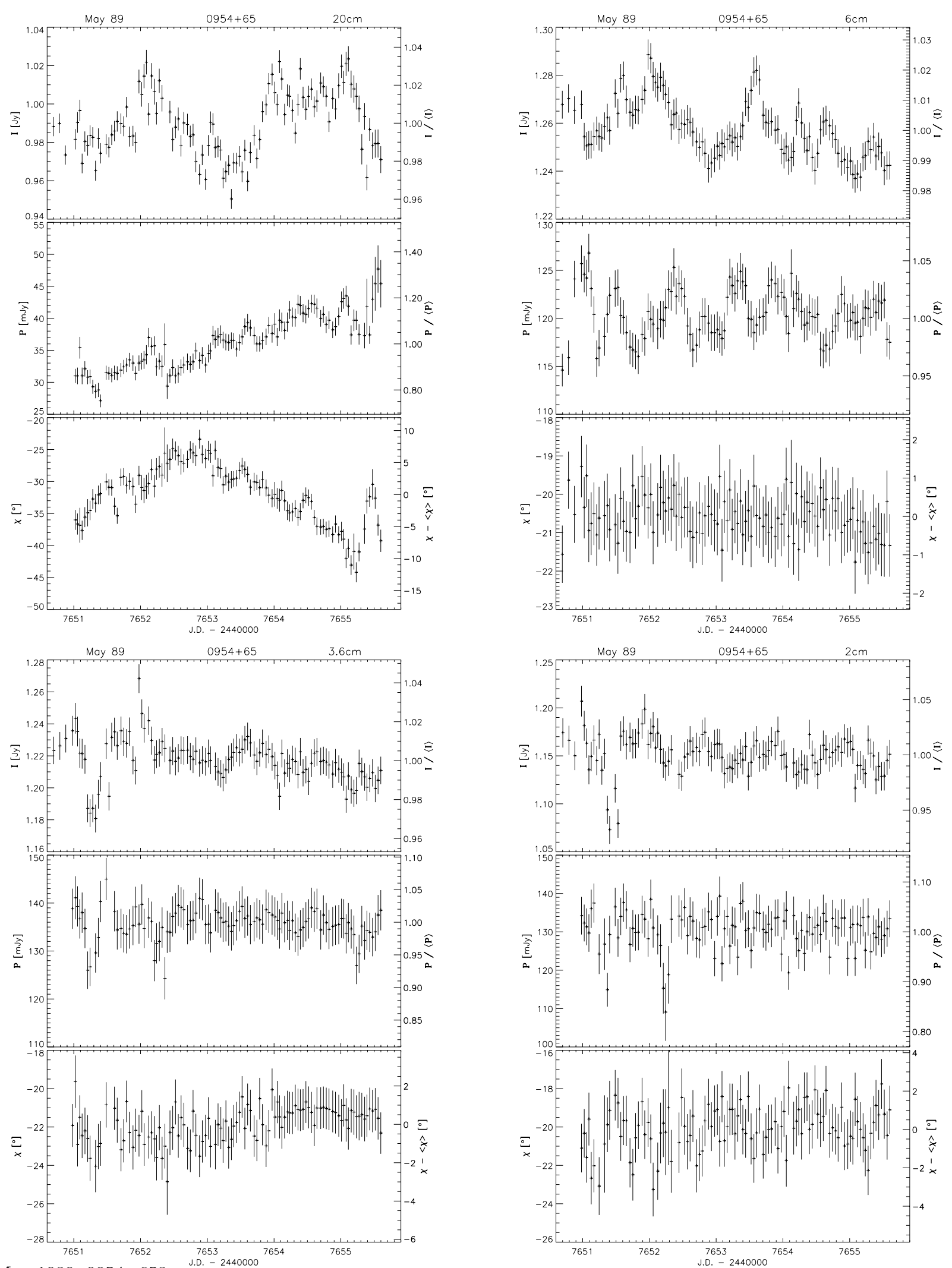

Fig. 5. May 1989; 0954+658 
A. Quirrenbach et al.: Intraday variability in compact extragalactic radio sources. I.
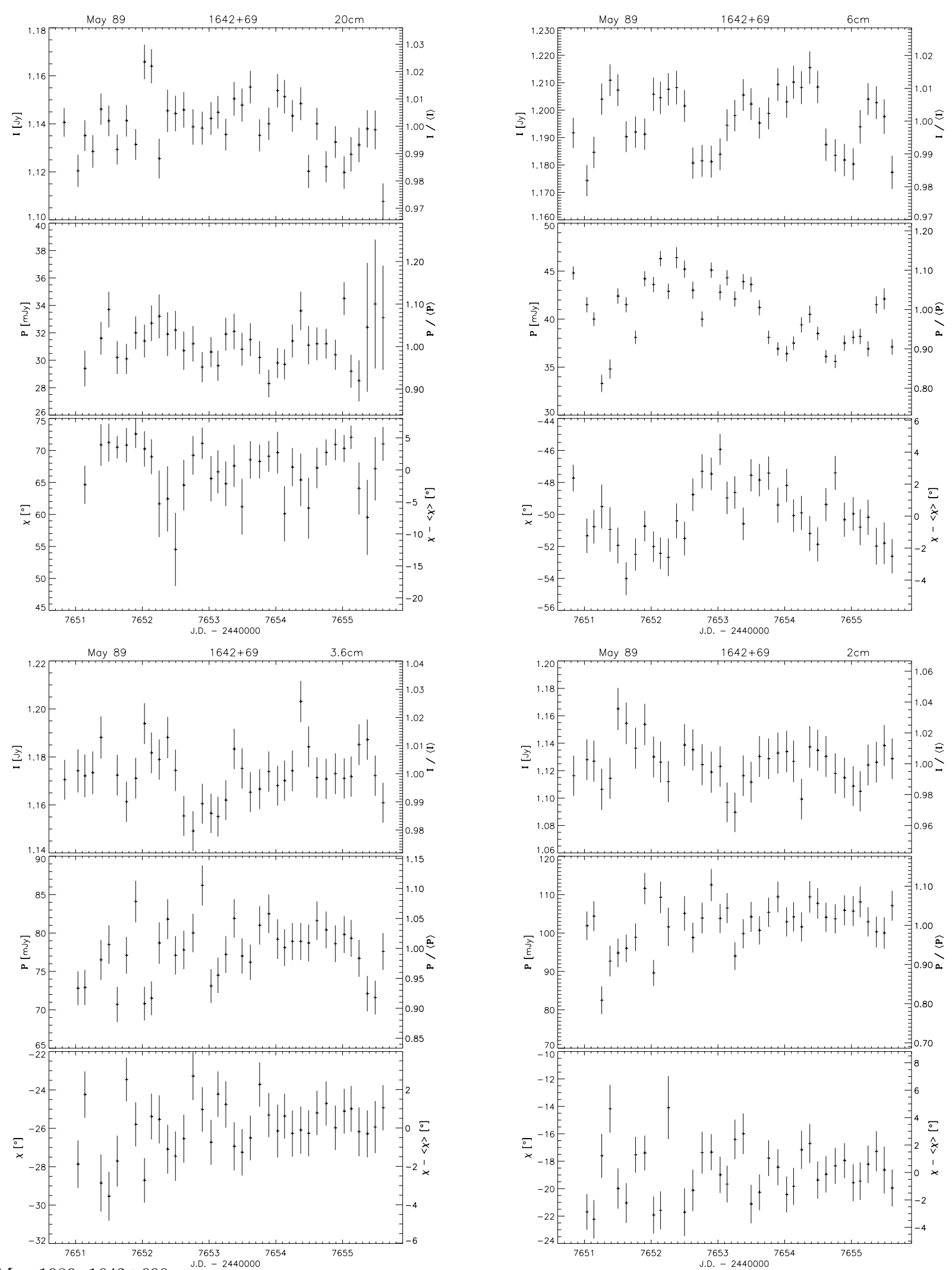

Fig. 6. May 1989; $1642+690$ 

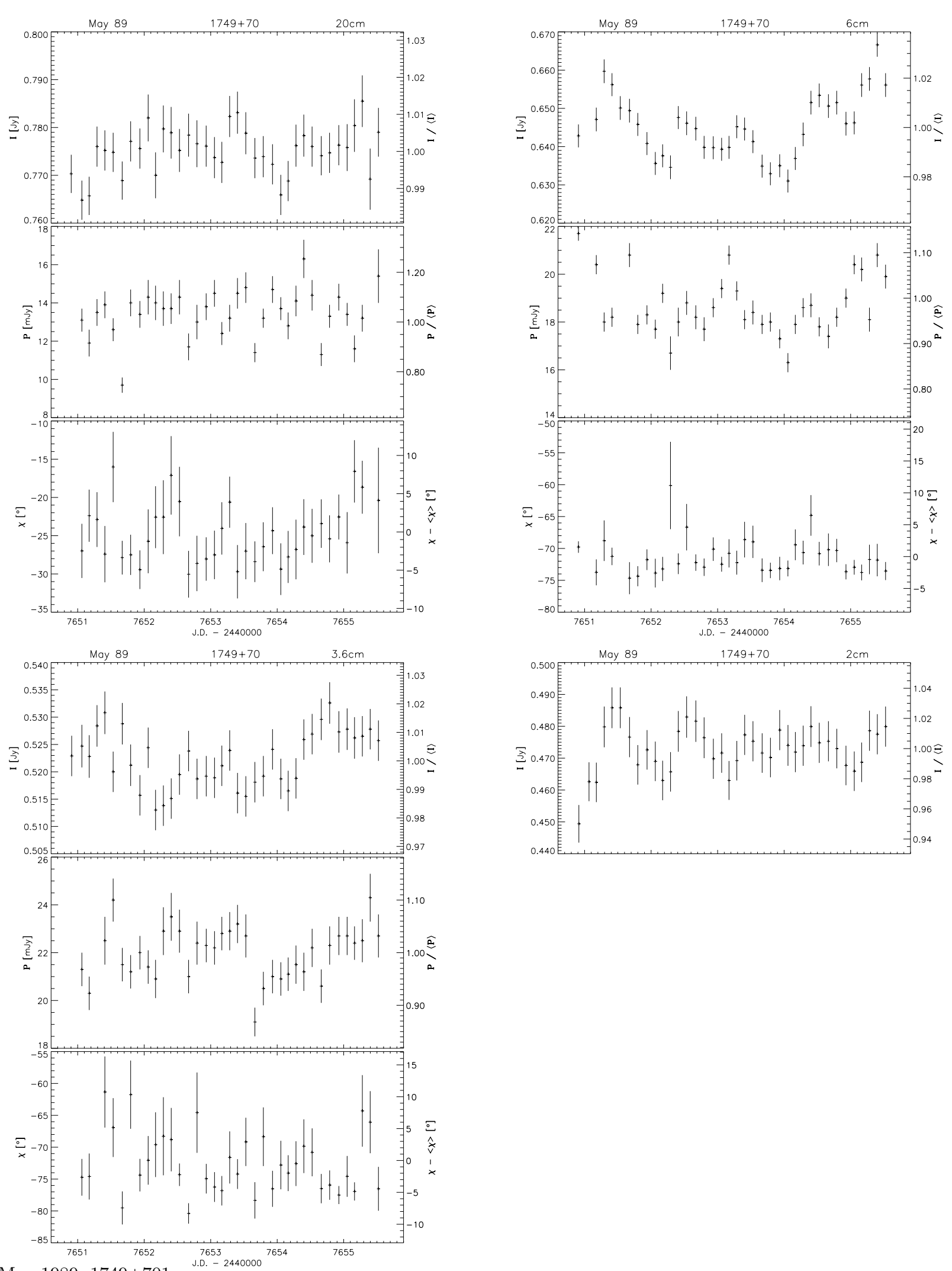

Fig. 7. May 1989; 1749+701 
A. Quirrenbach et al.: Intraday variability in compact extragalactic radio sources. I.
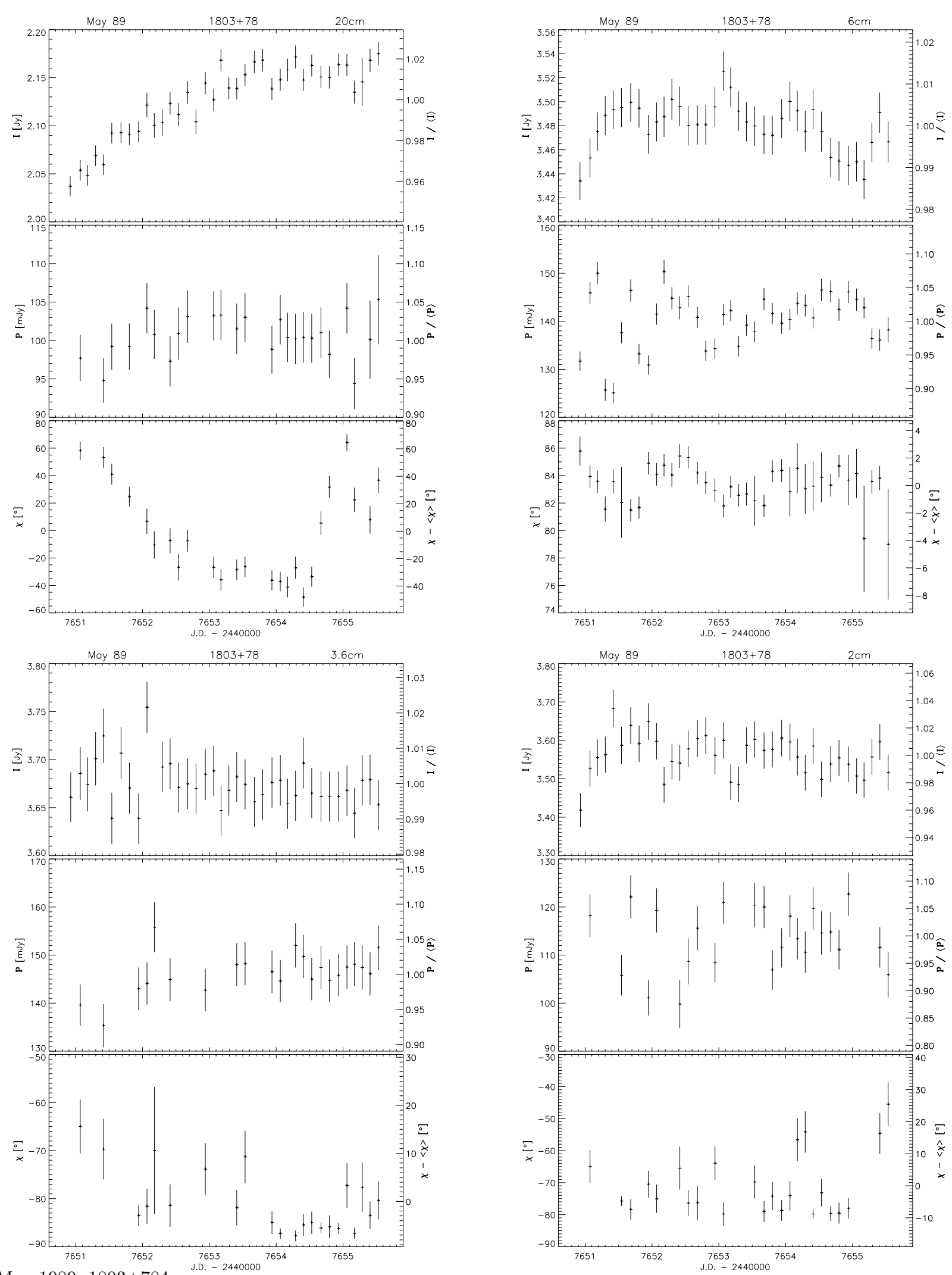

Fig. 8. May 1989; 1803+784 

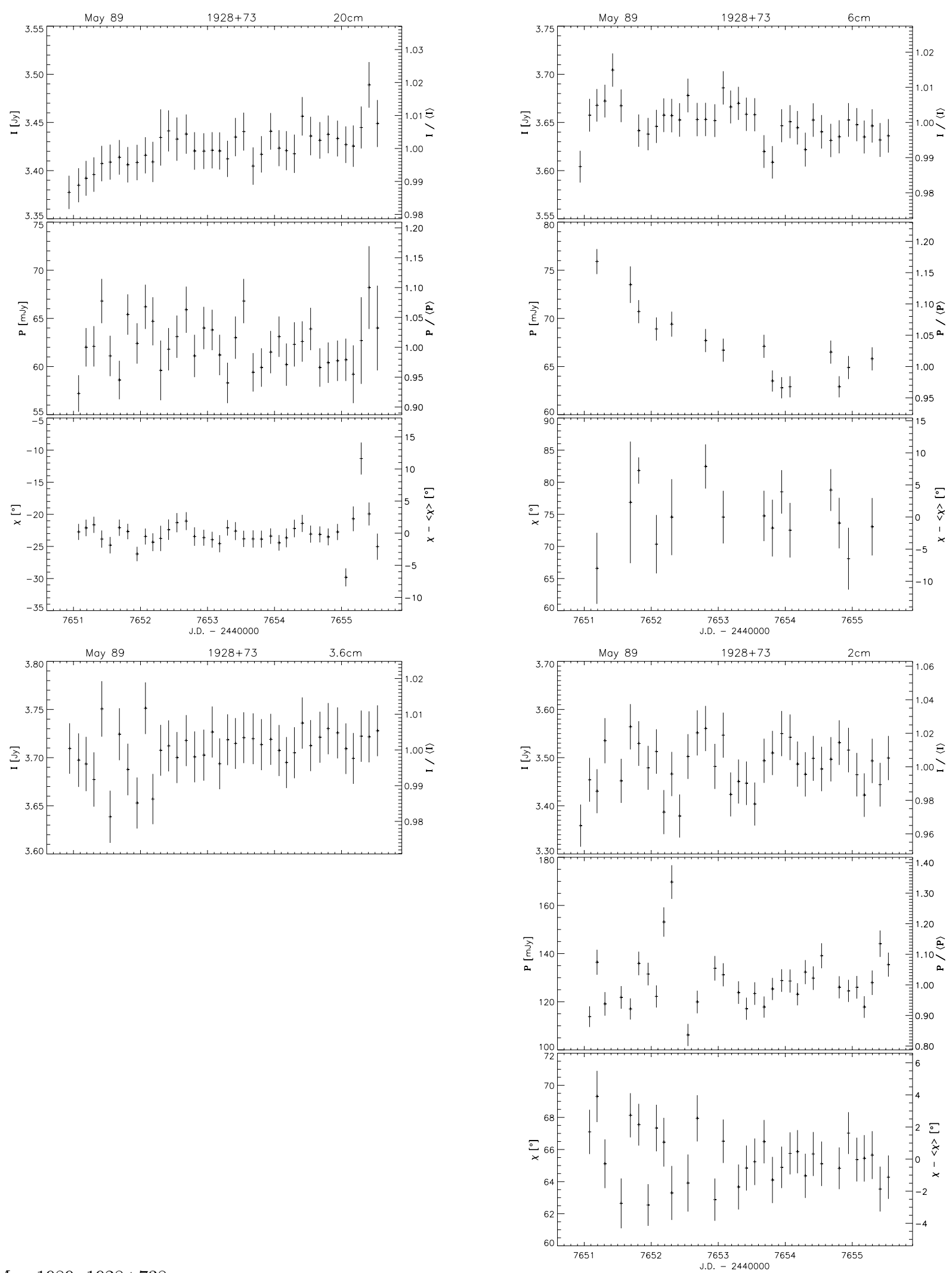

Fig. 9. May 1989; 1928+738 
A. Quirrenbach et al.: Intraday variability in compact extragalactic radio sources. I.
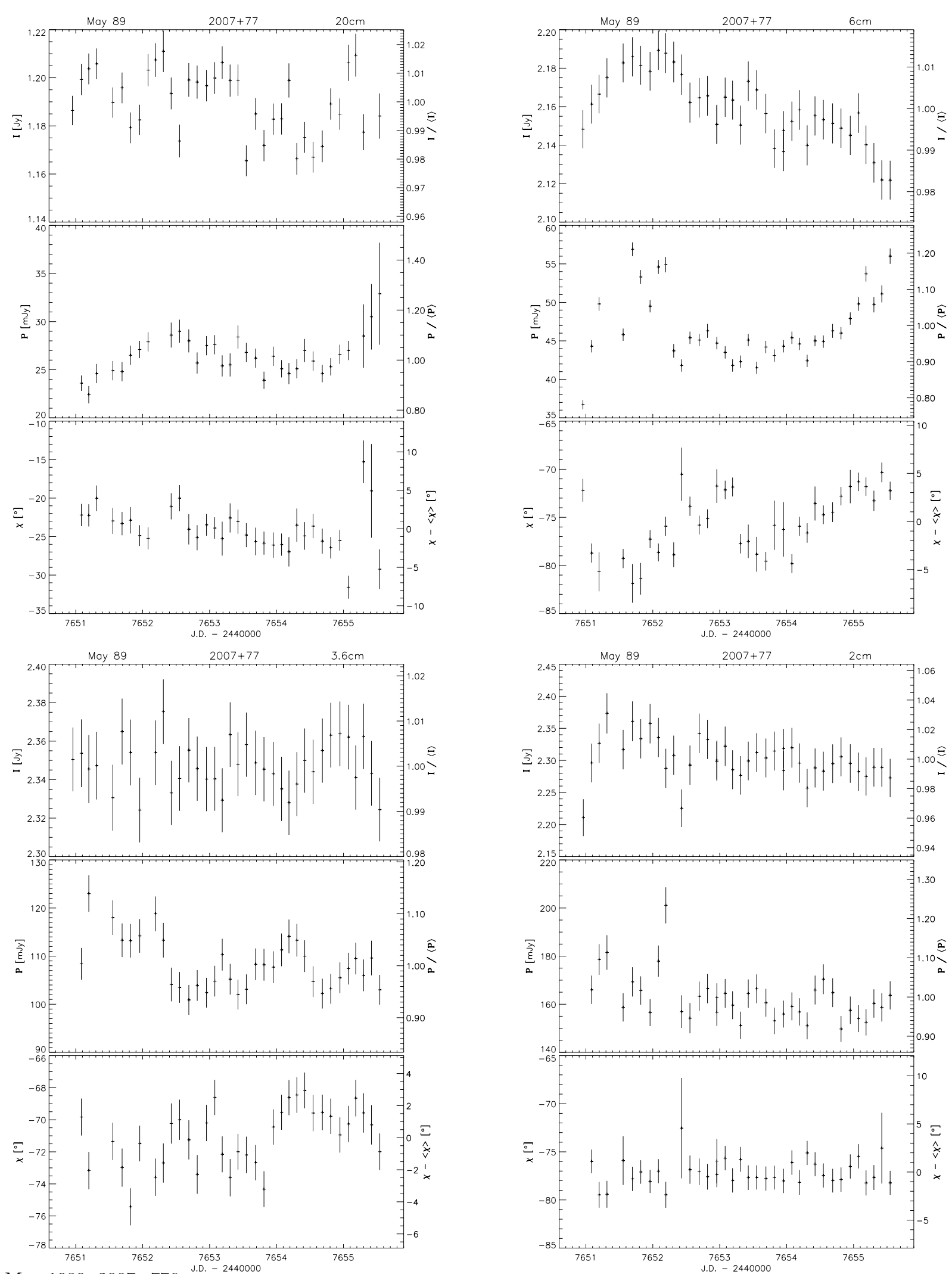

Fig. 10. May 1989; 2007+776 

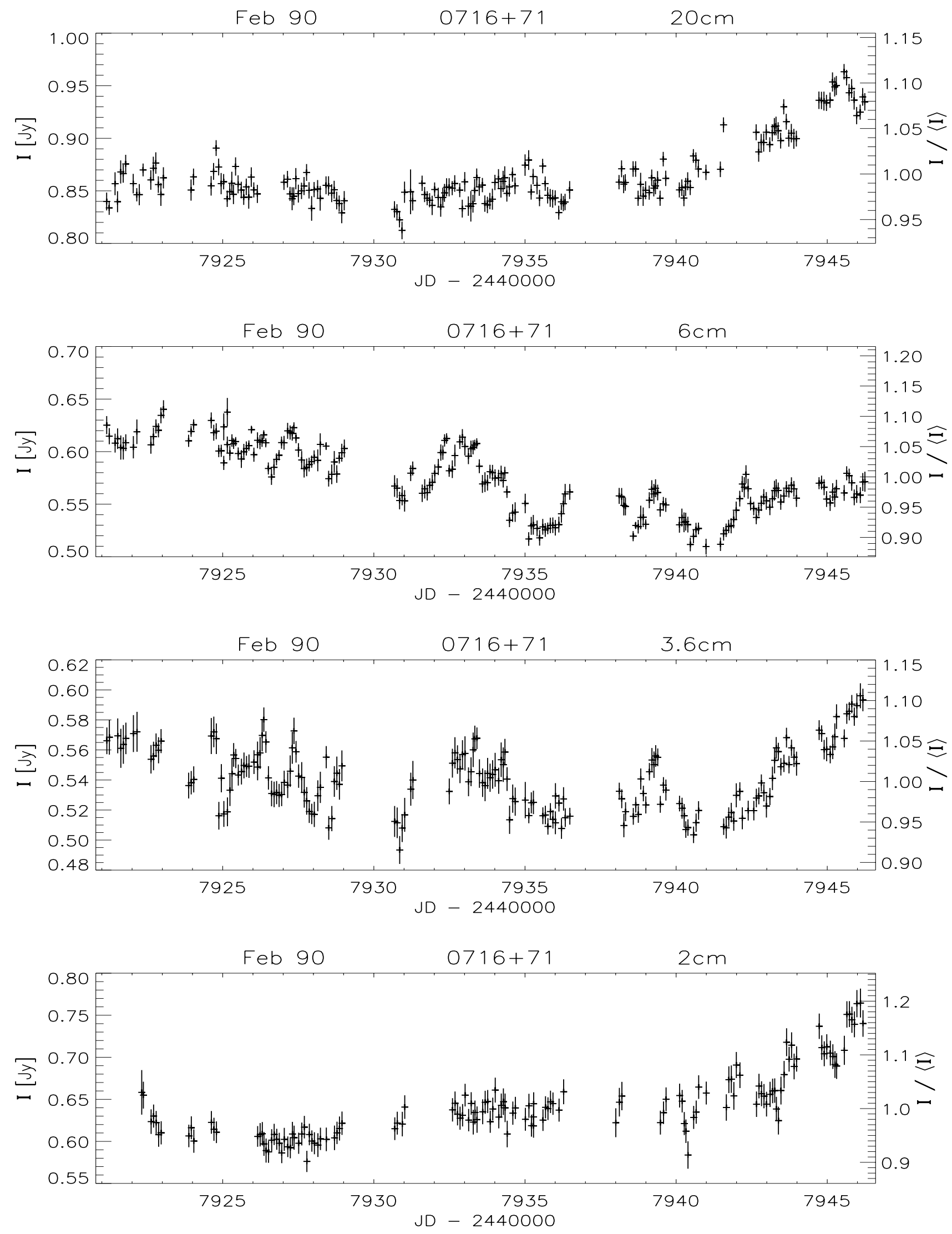

Fig. 11. February 1990: 0716+714 

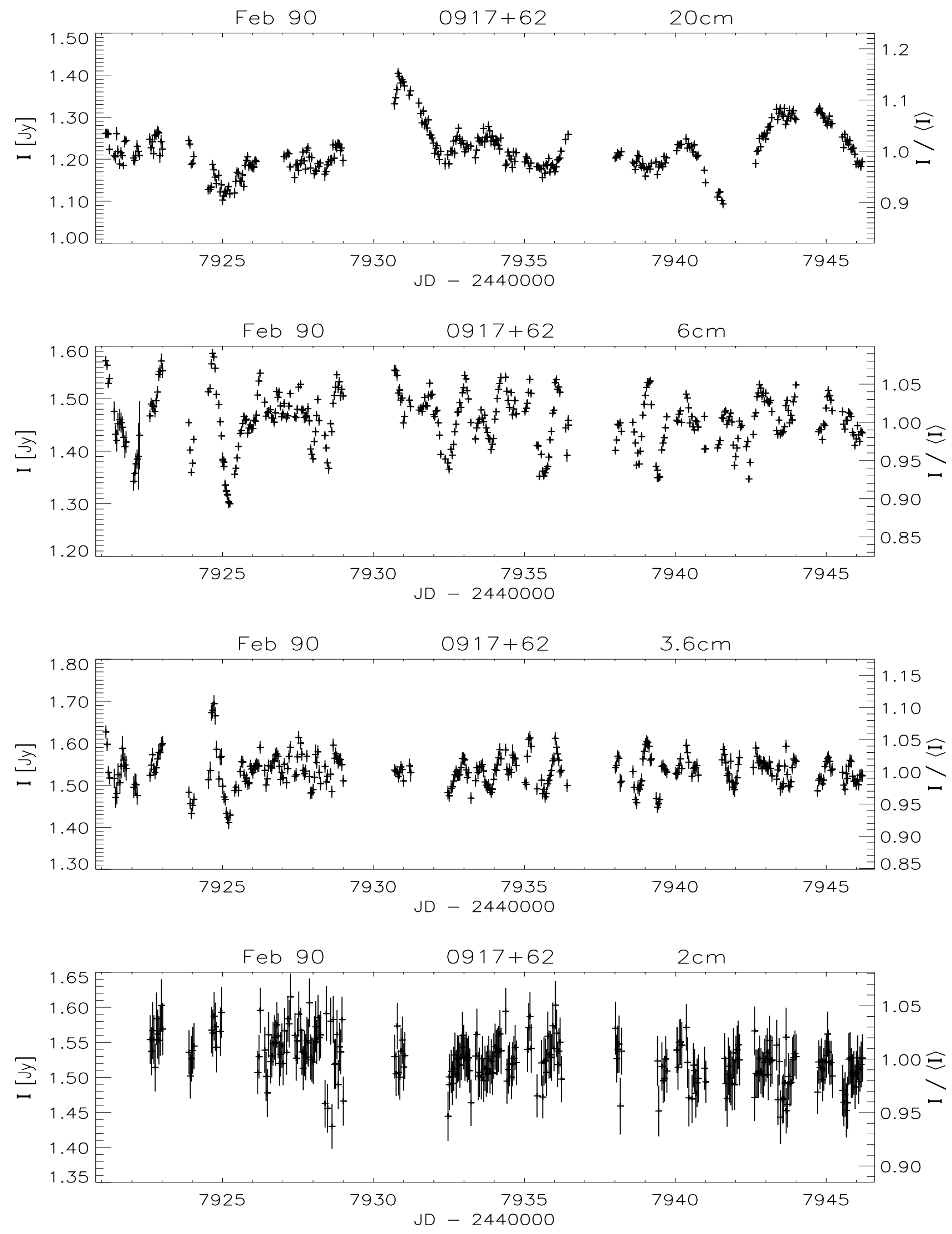

Fig. 12. February 1990: 0917+624 

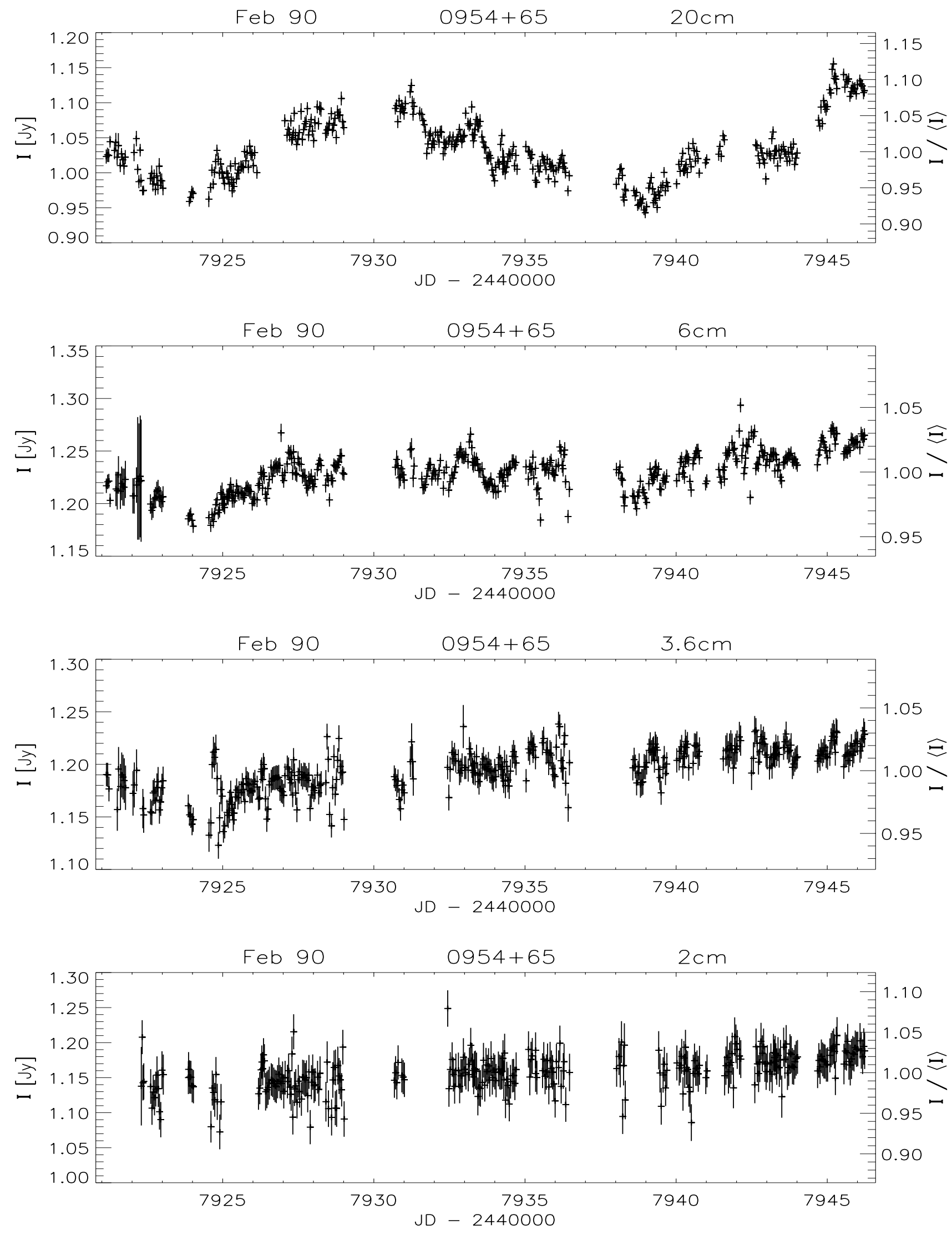

Fig. 13. February 1990: 0954+658 

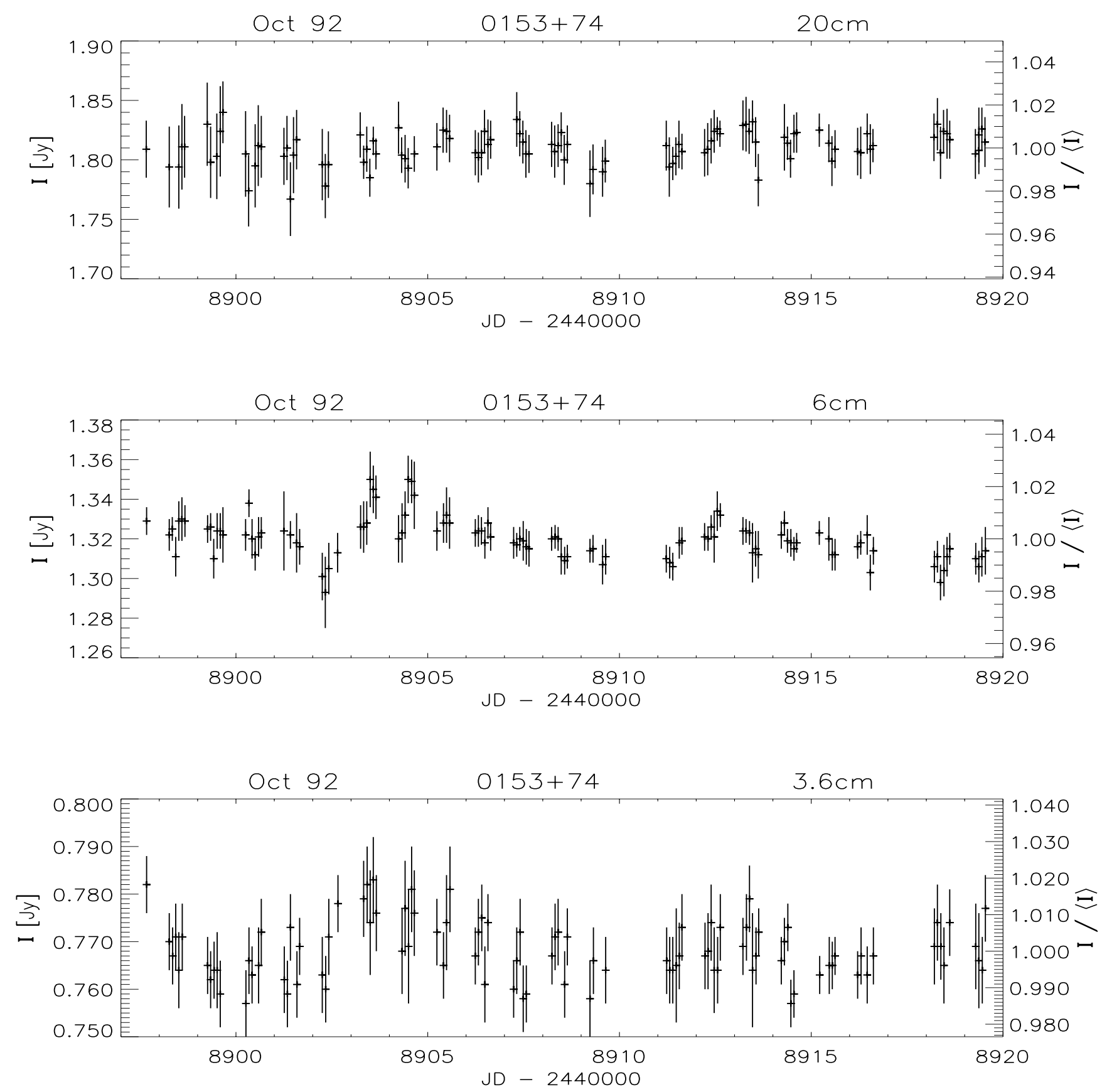

Fig. 14. October 1992: 0153+744 

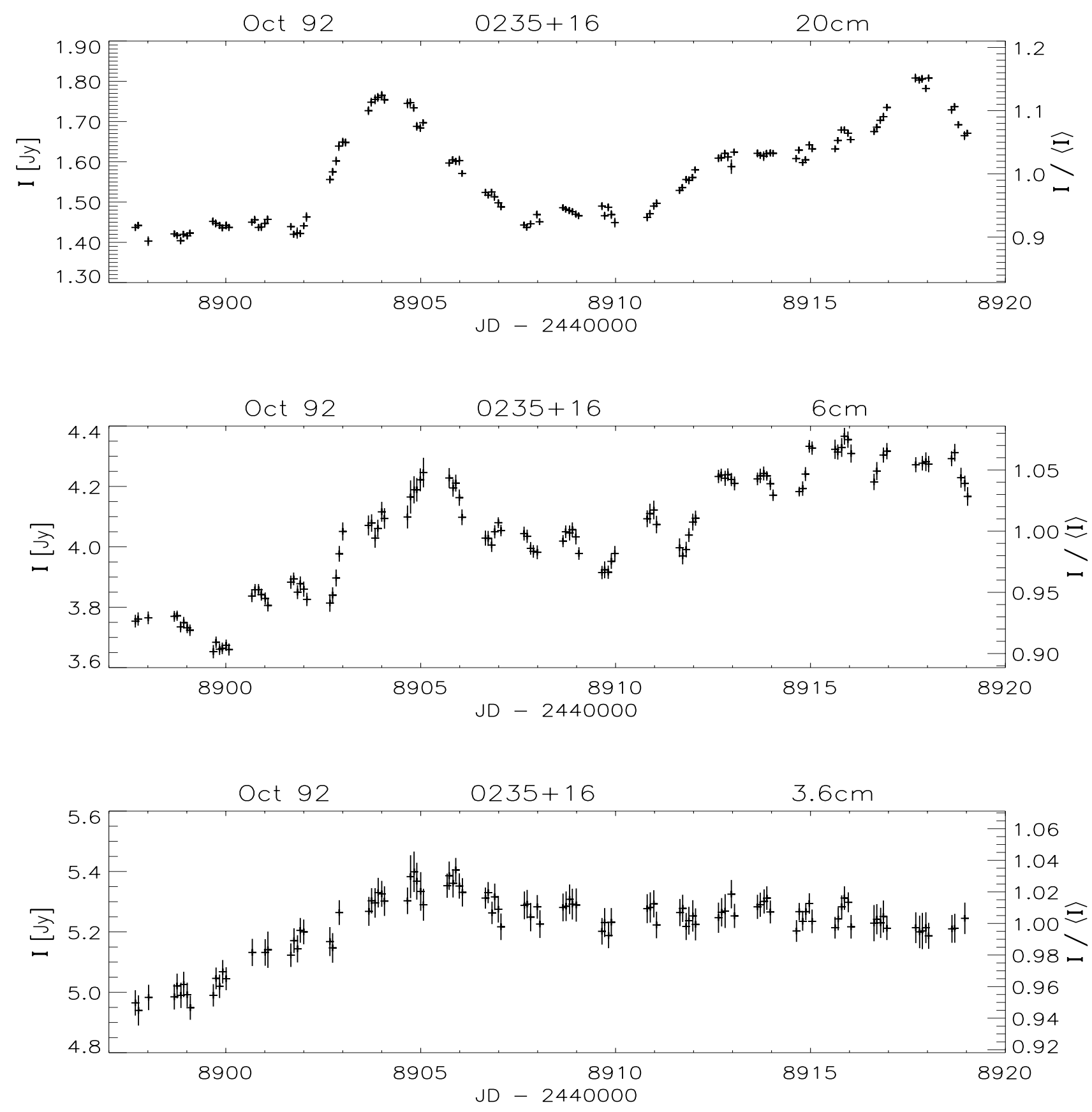

Fig. 15. October 1992: 0235+164 

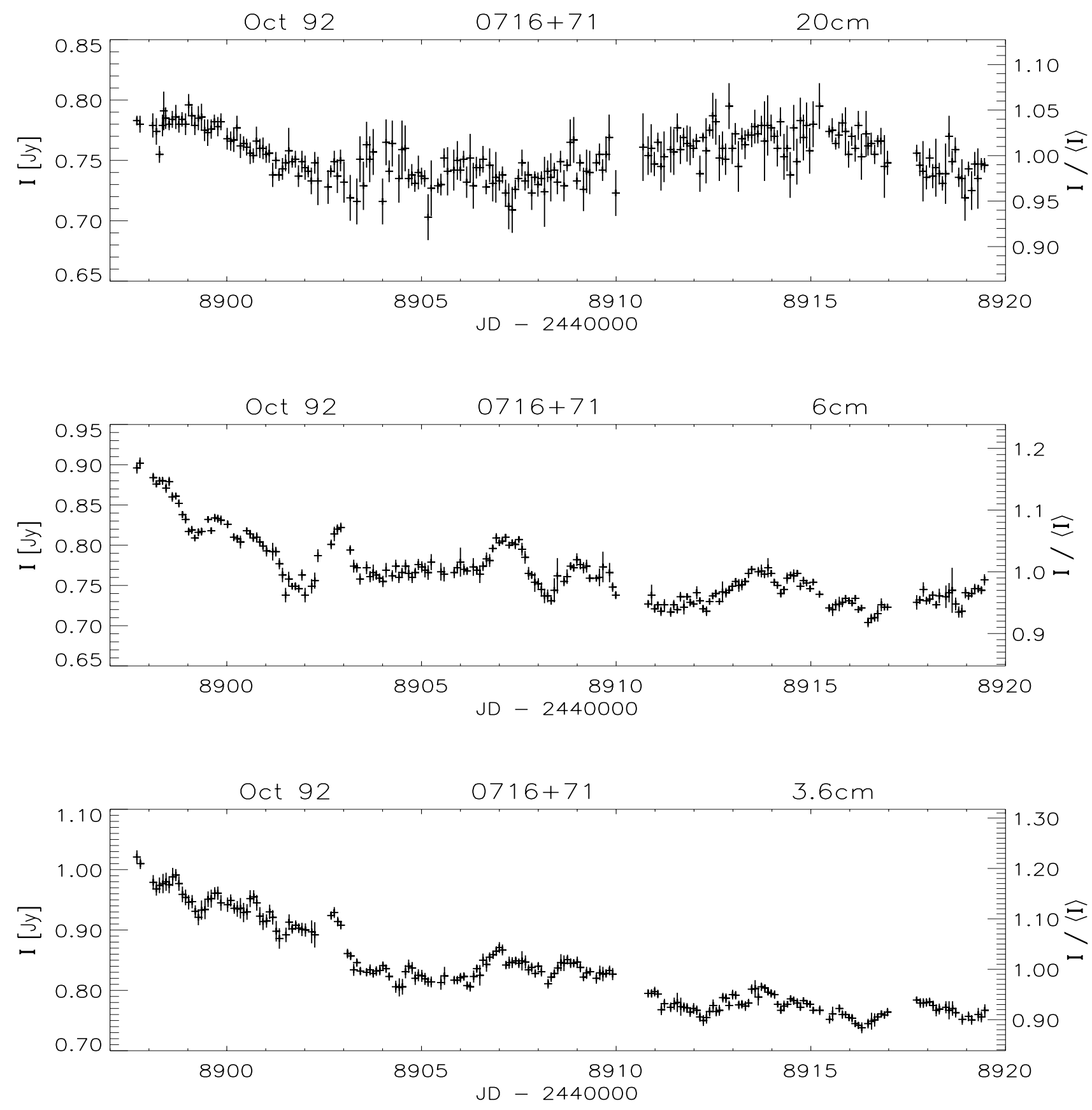

Fig. 16. October 1992: $0716+714$ 

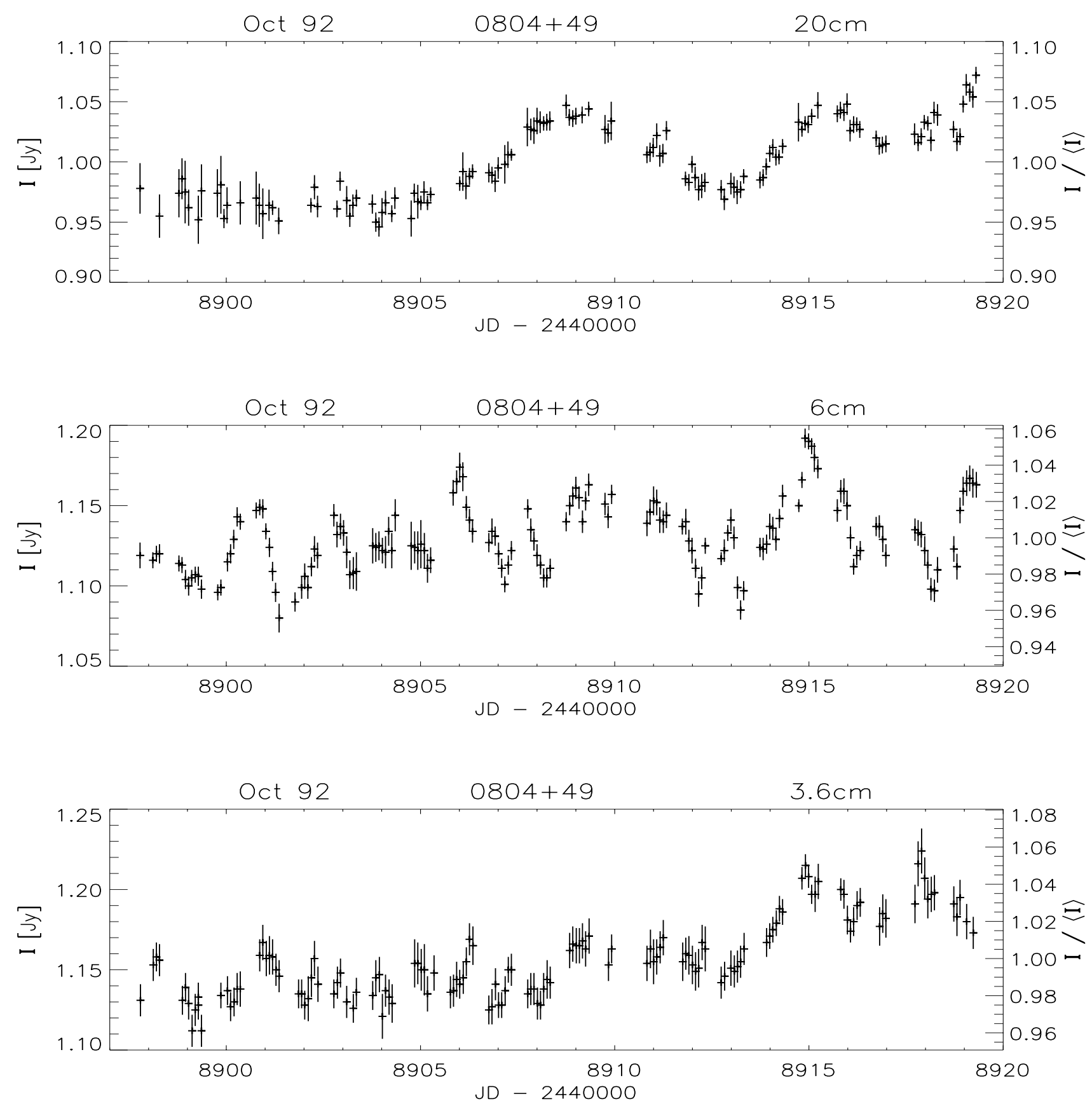

Fig. 17. October 1992: 0804+499 

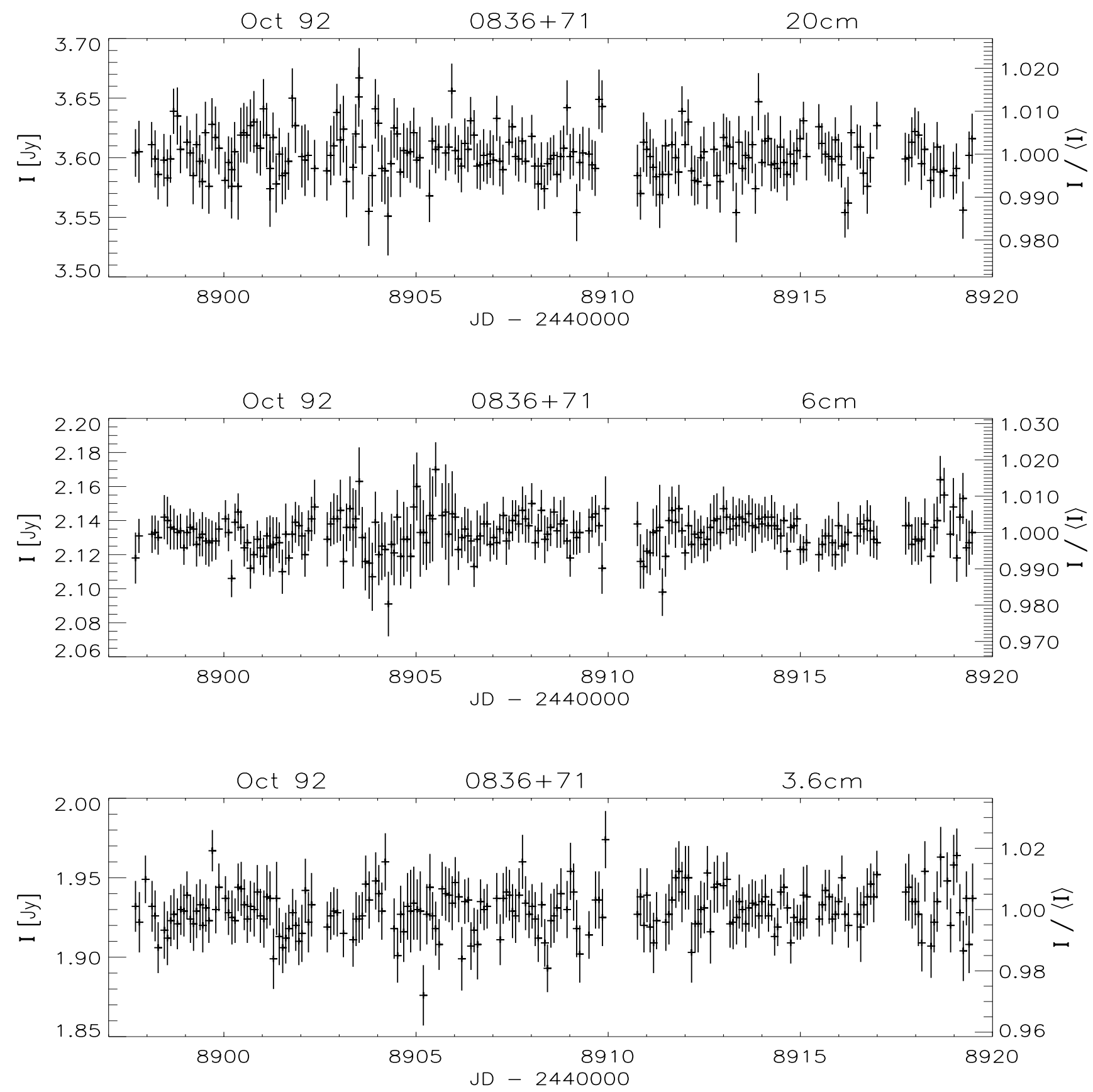

Fig. 18. October 1992: $0836+710$ 

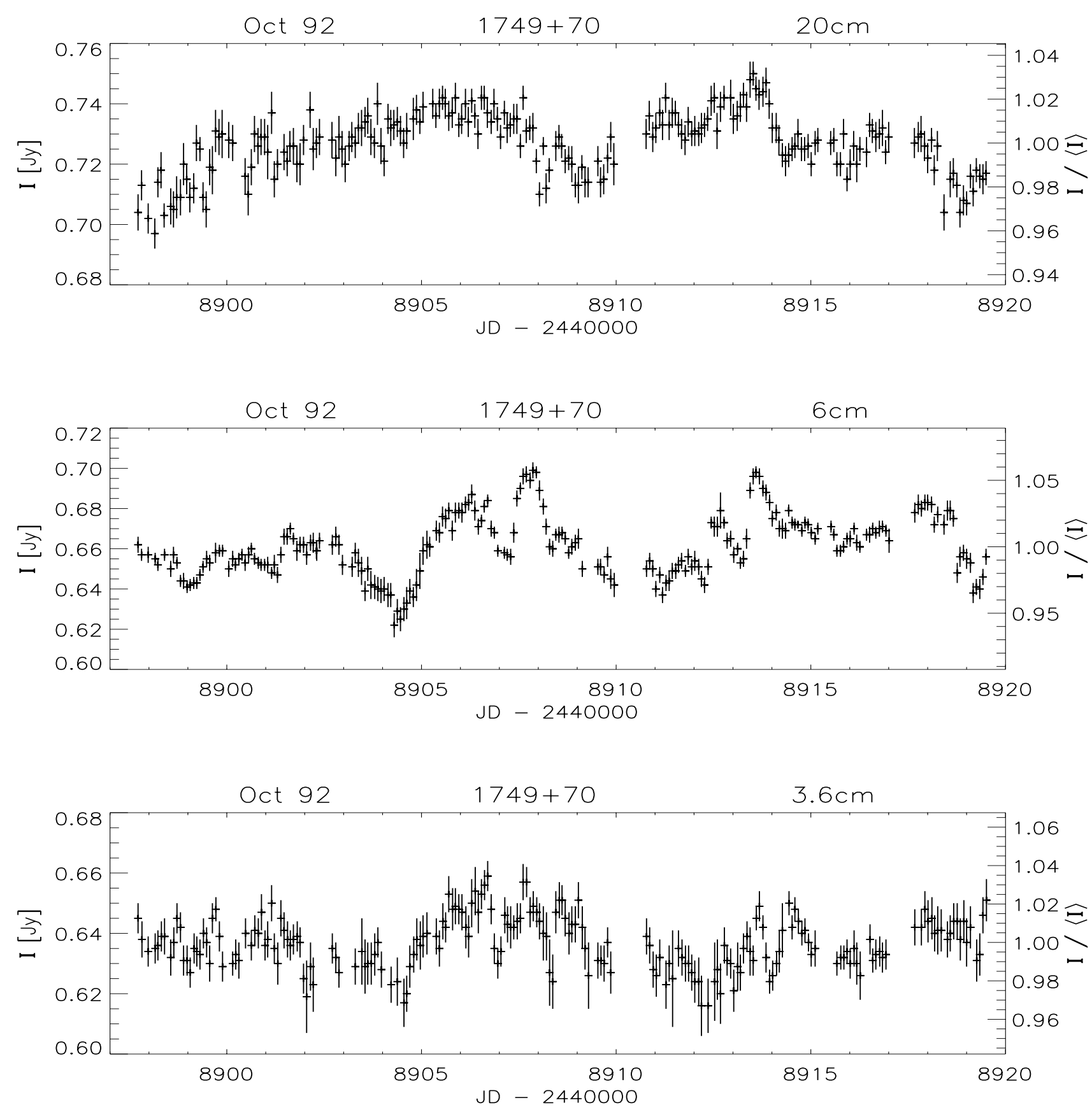

Fig. 19. October 1992: 1749+701 

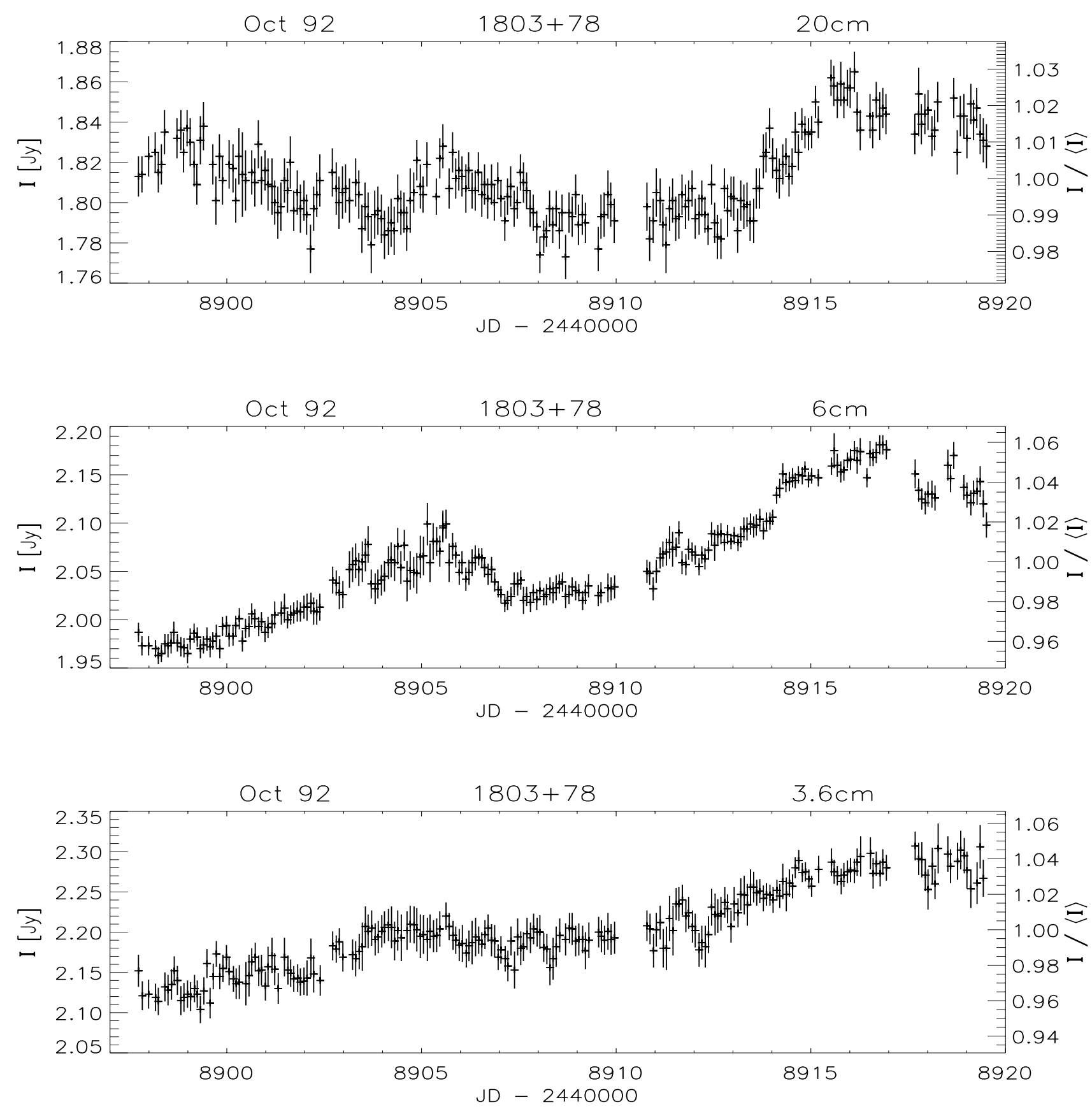

Fig. 20. October 1992: $1803+784$ 

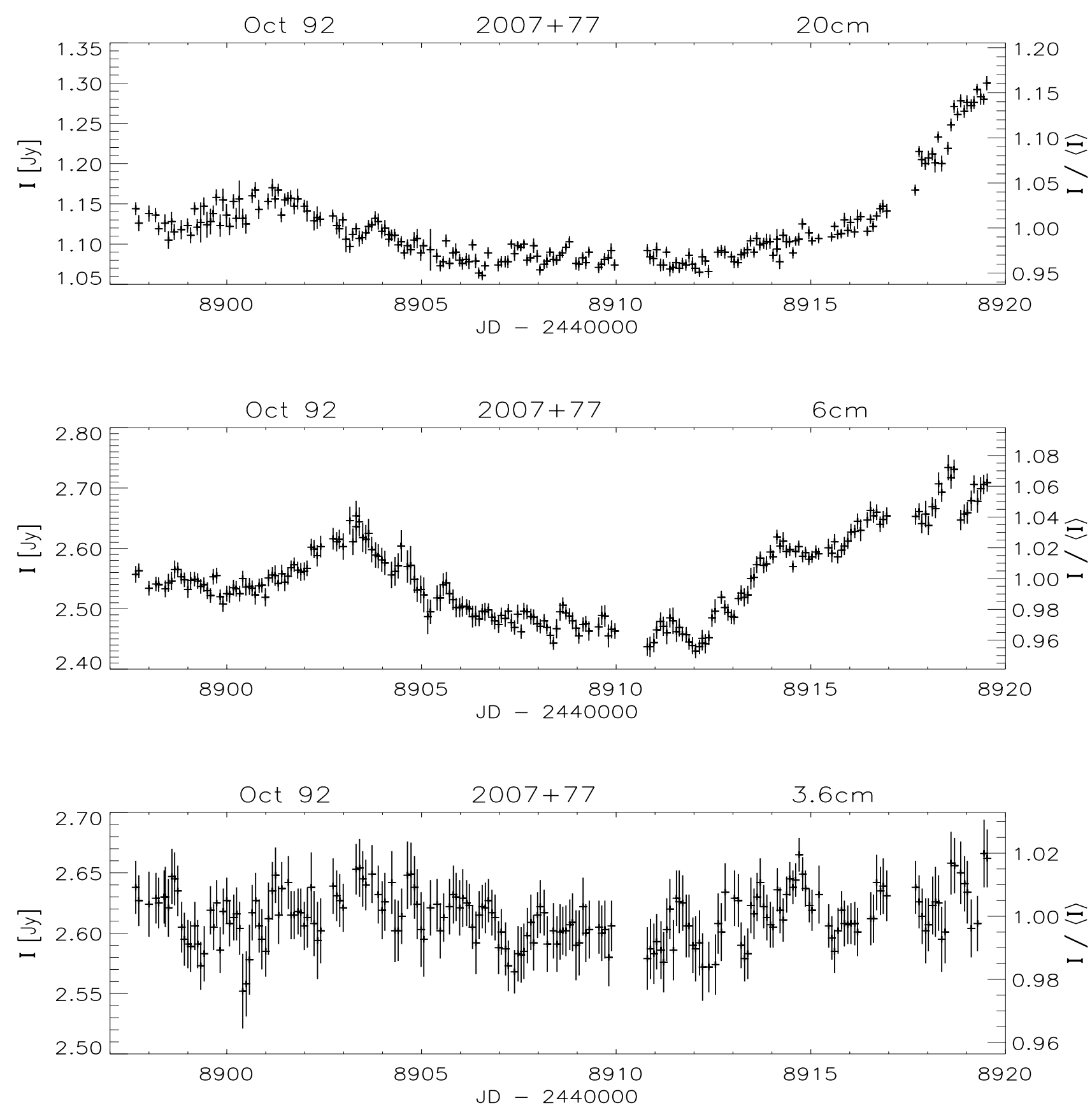

Fig. 21. October 1992: $2007+776$ 
A. Quirrenbach et al.: Intraday variability in compact extragalactic radio sources. I.

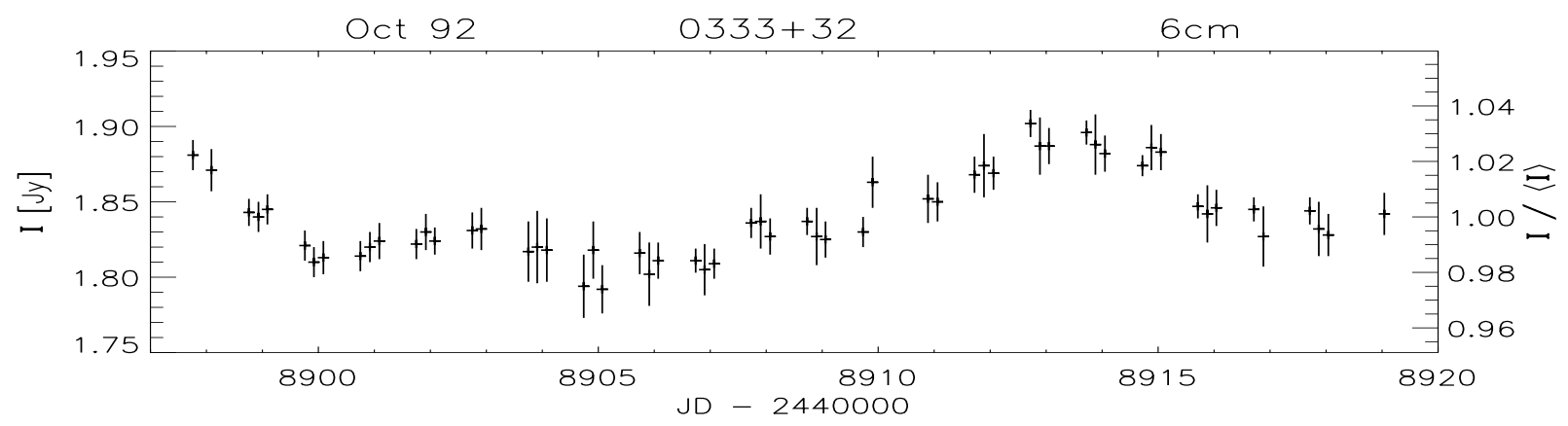

Fig. 22. October 1992: 0333+321

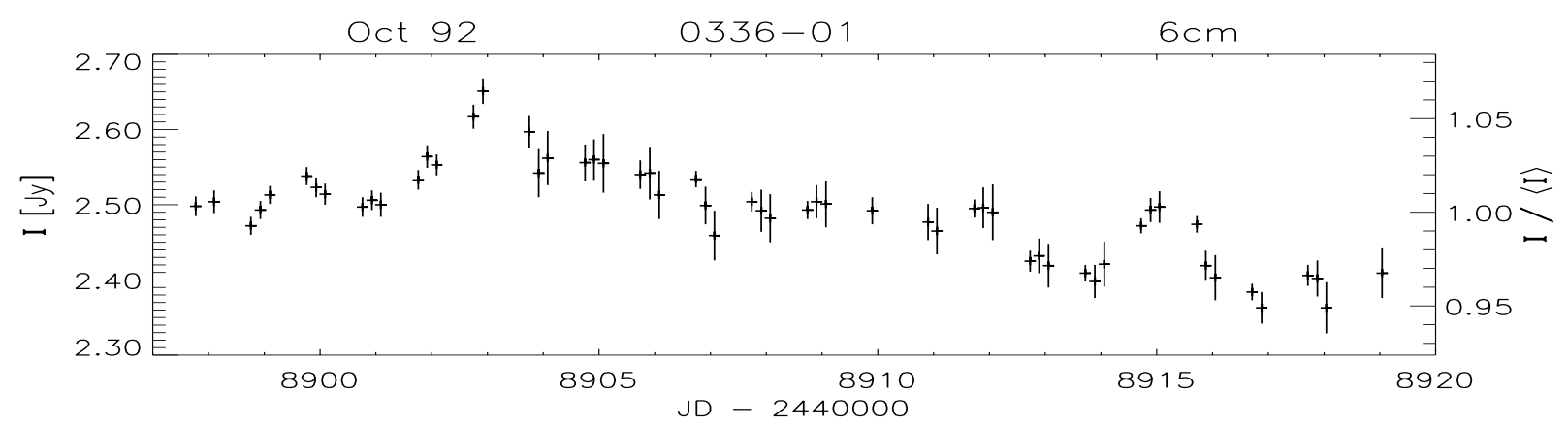

Fig. 23. October 1992: 0336-019
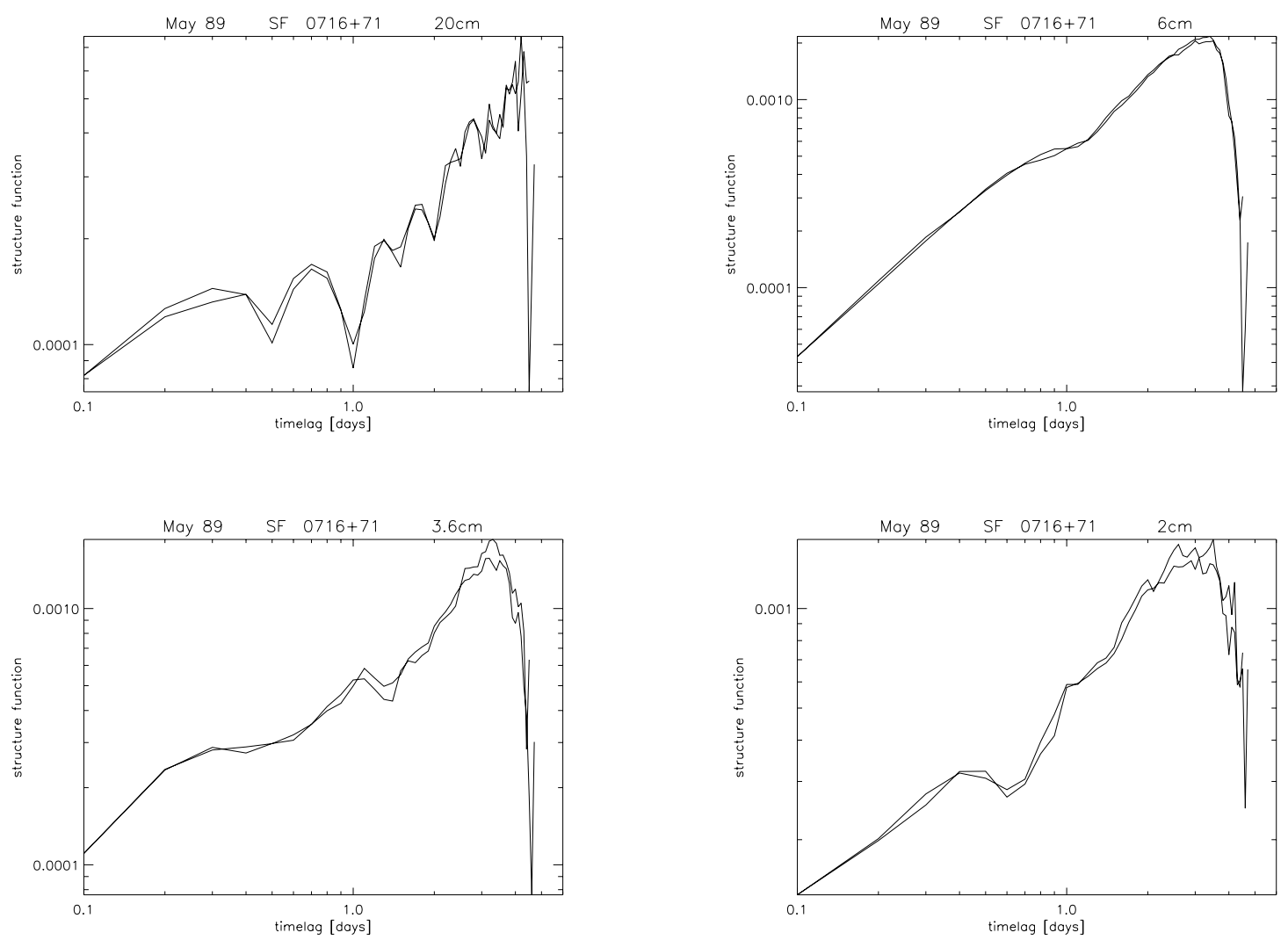

Fig. 24. Structure functions May 1989;0716+714 
A. Quirrenbach et al.: Intraday variability in compact extragalactic radio sources. I.
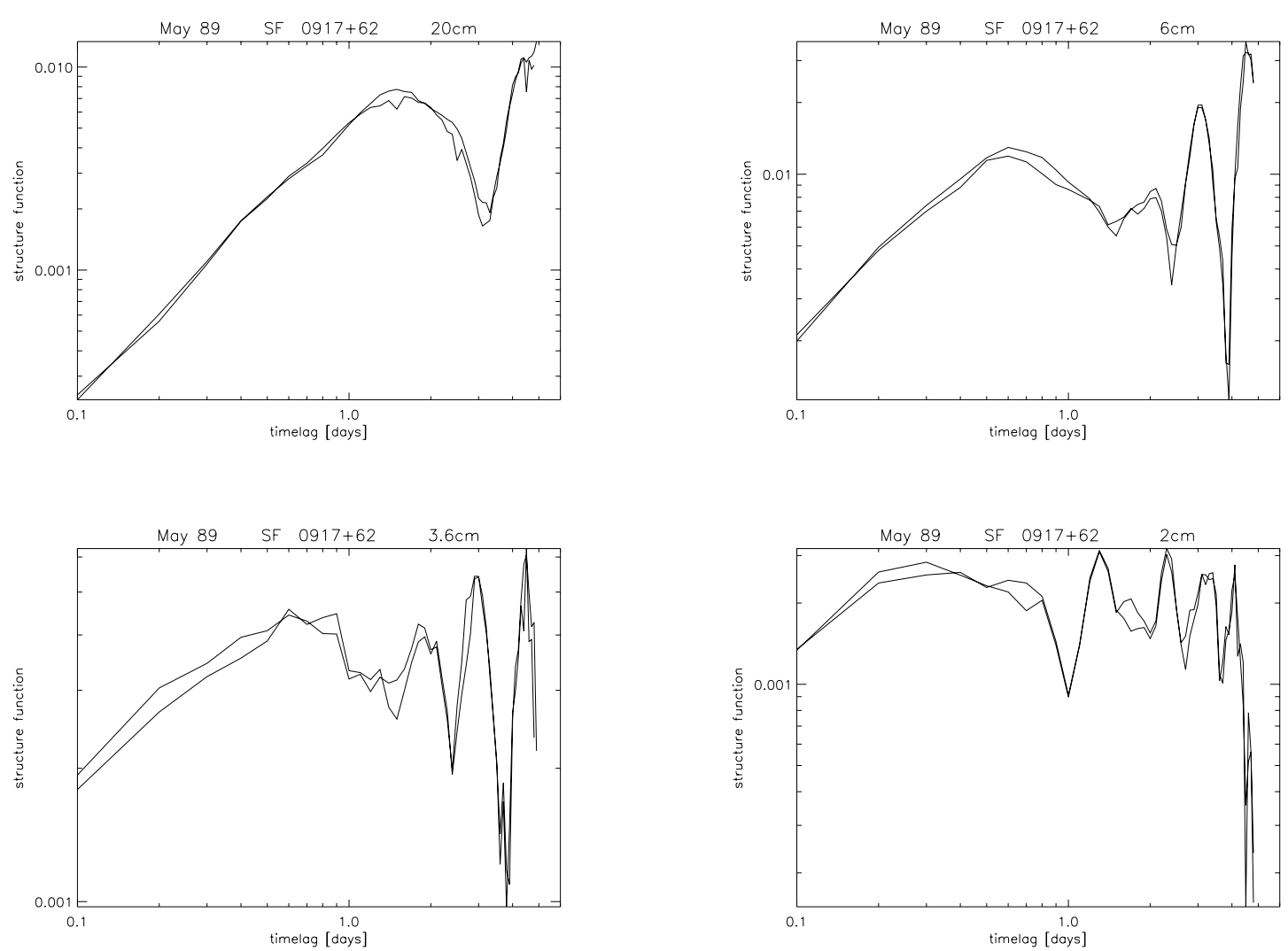

Fig. 25. Structure functions May 1989; 0917+624
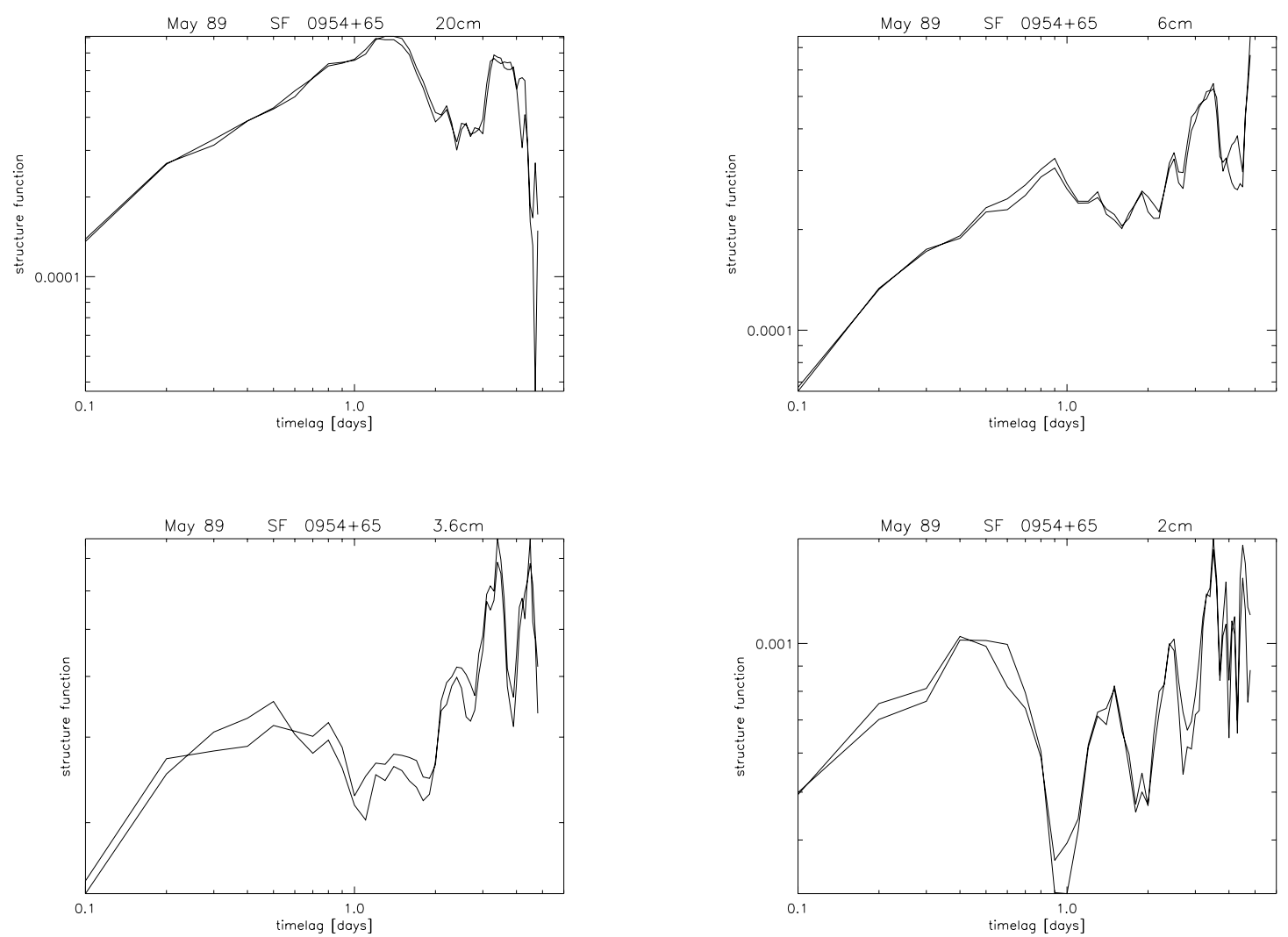

Fig. 26. Structure functions May 1989; 0954+658 

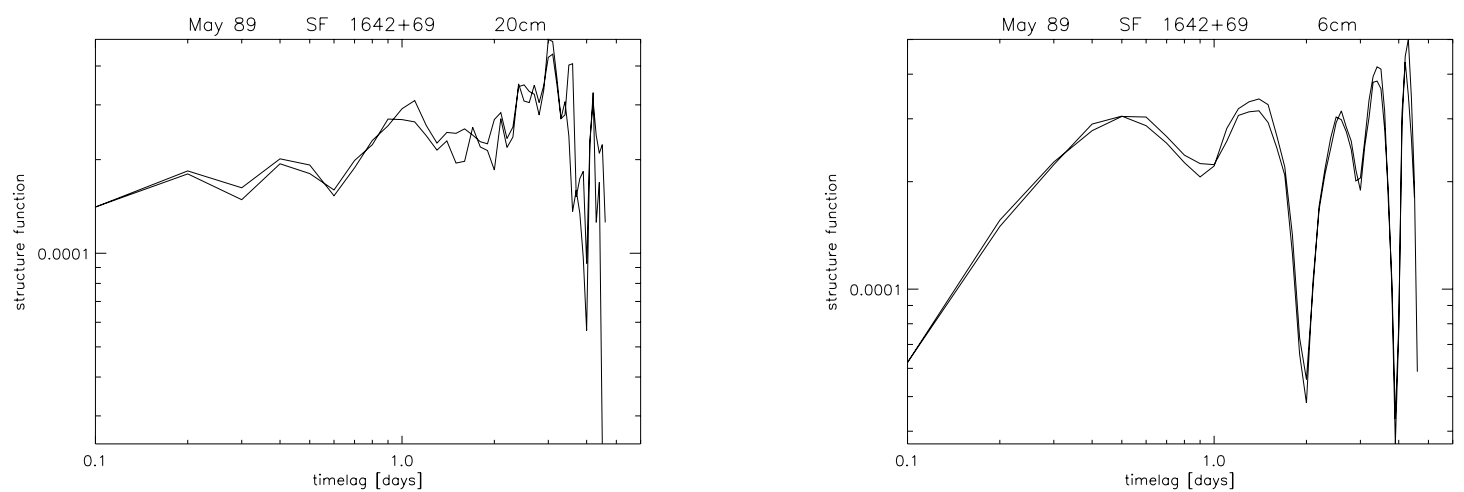

Fig. 27. Structure functions May 1989; 1642+690

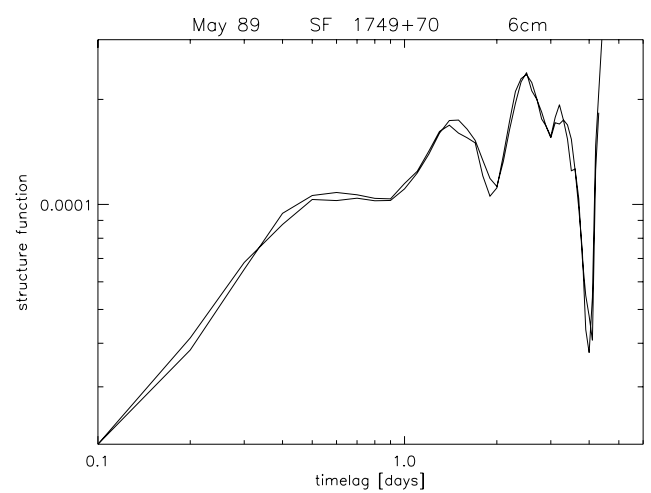

Fig. 28. Structure function May 1989; 1749+701

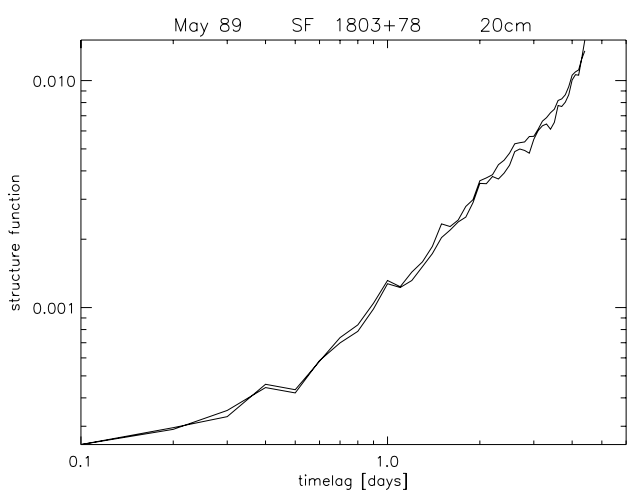

Fig. 29. Structure function May 1989; 1803+784 

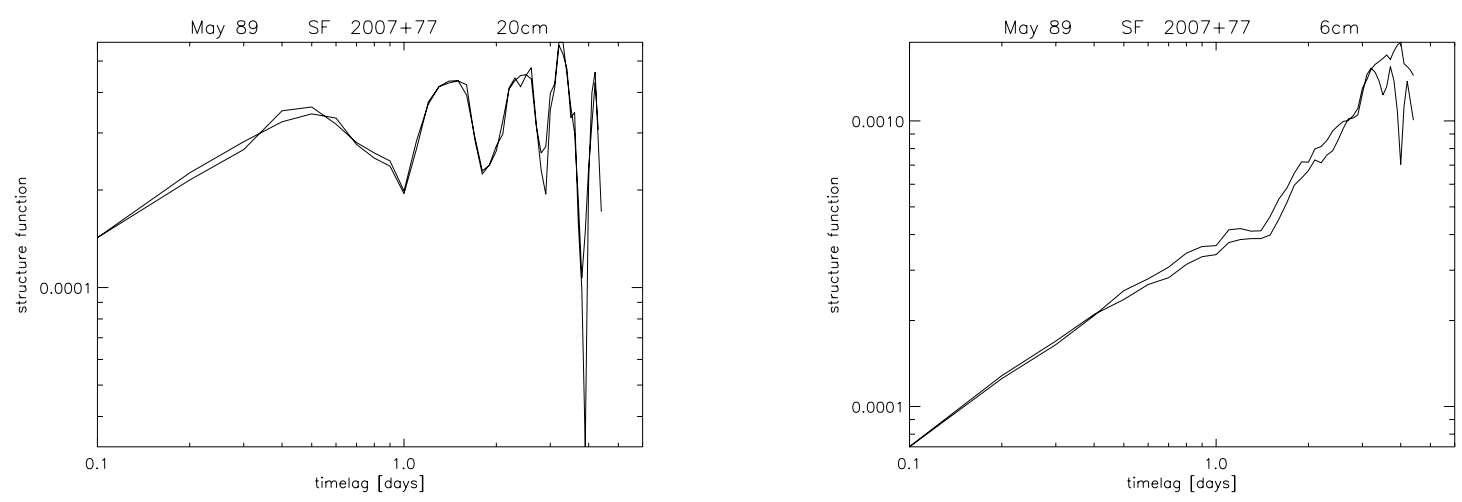

Fig. 30. Structure functions May 1989; 2007+777
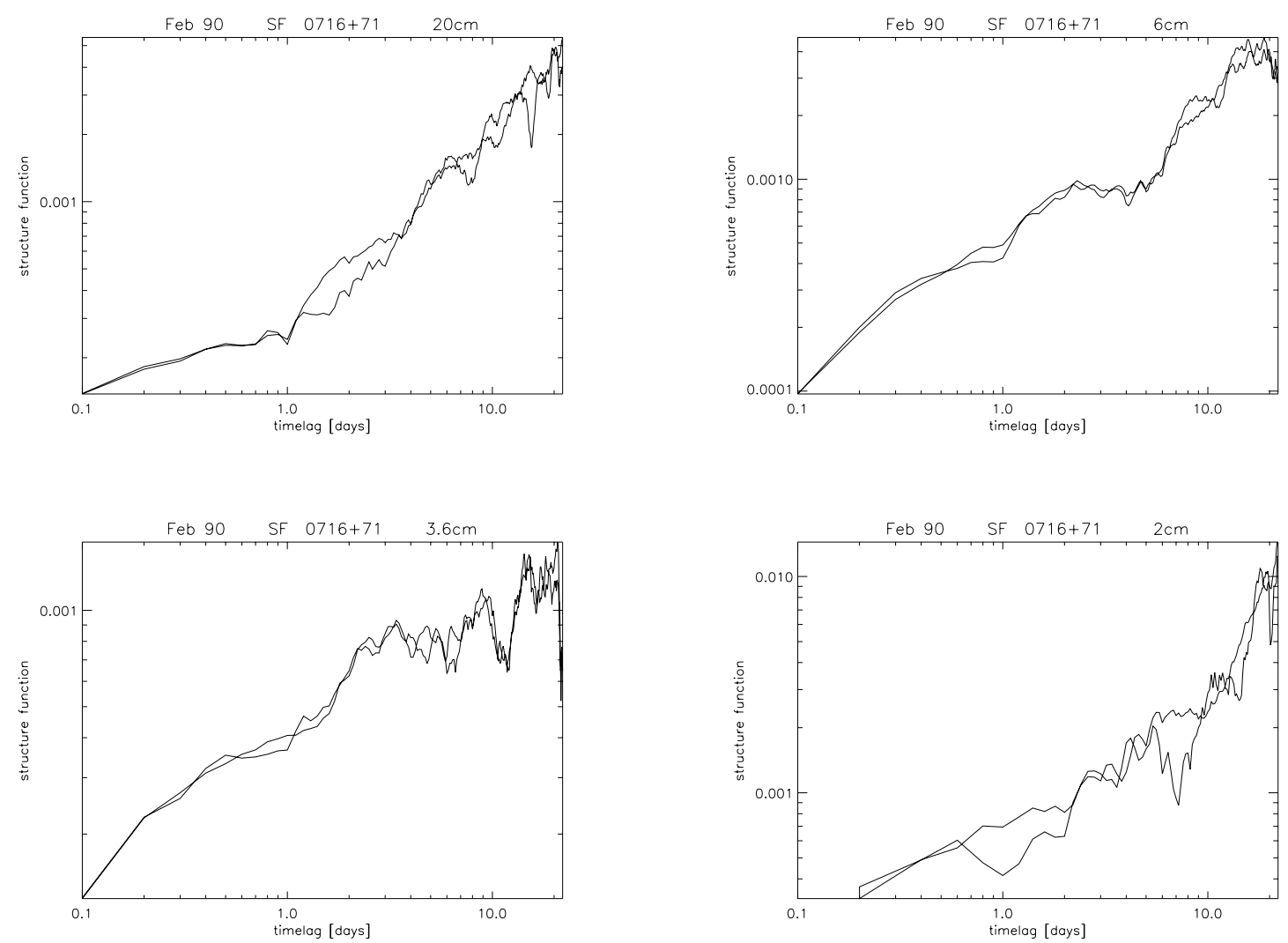

Fig. 31. Structure function February 1990; 0716+714 
A. Quirrenbach et al.: Intraday variability in compact extragalactic radio sources. I.
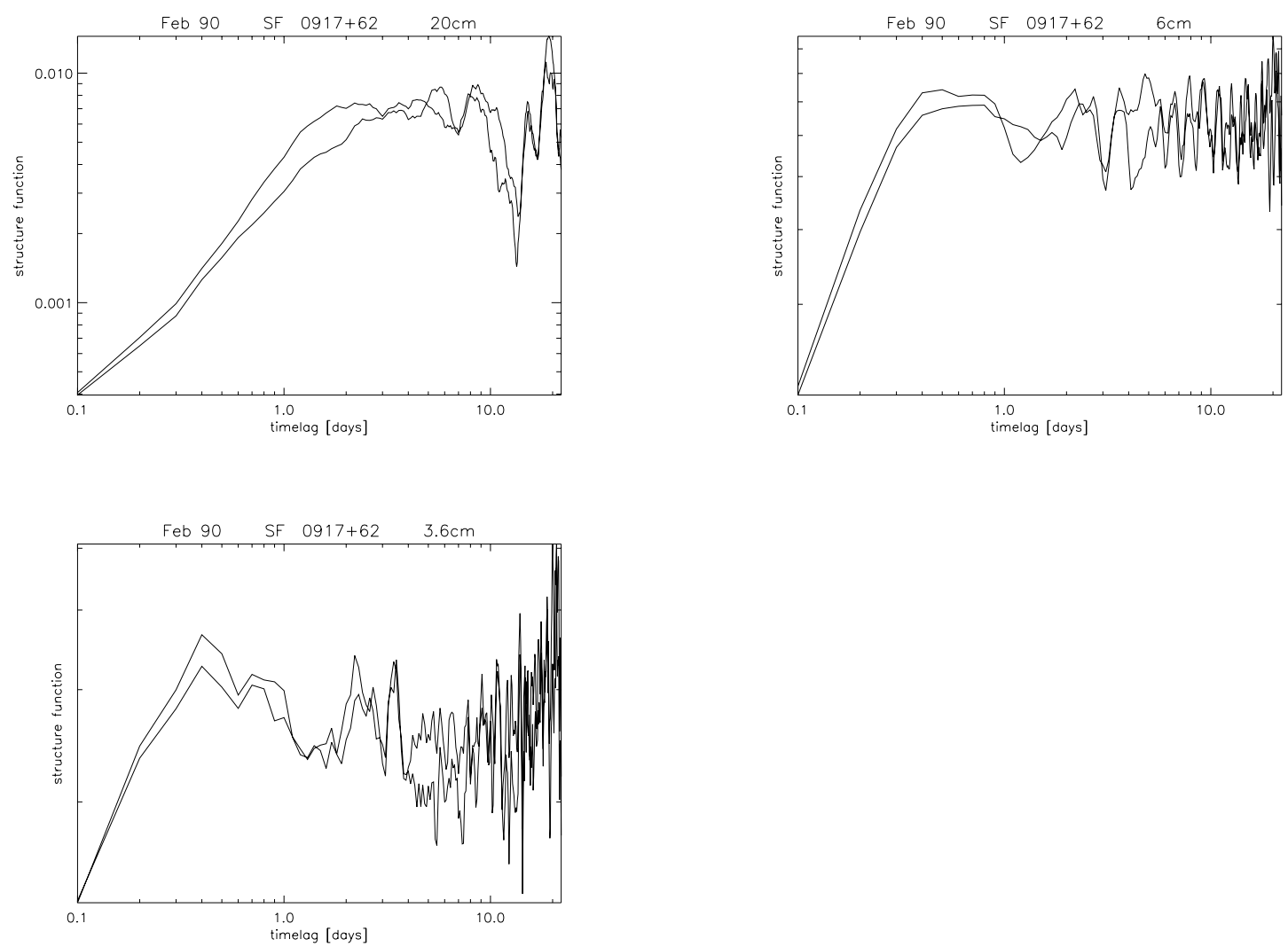

Fig. 32. Structure functions February 1990; 0917+624
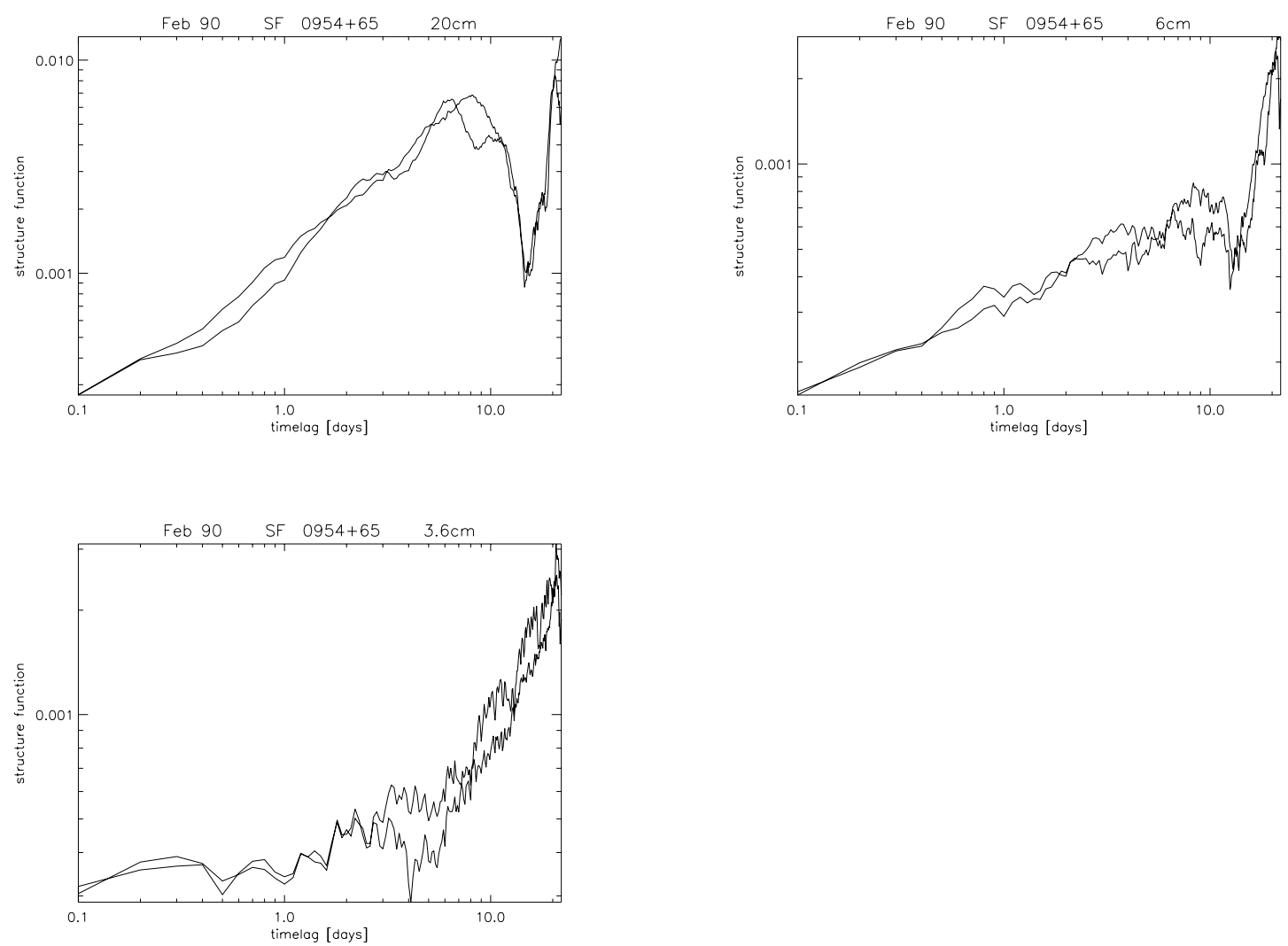

Fig. 33. Structure functions February 1990; 0954+658 
A. Quirrenbach et al.: Intraday variability in compact extragalactic radio sources. I.
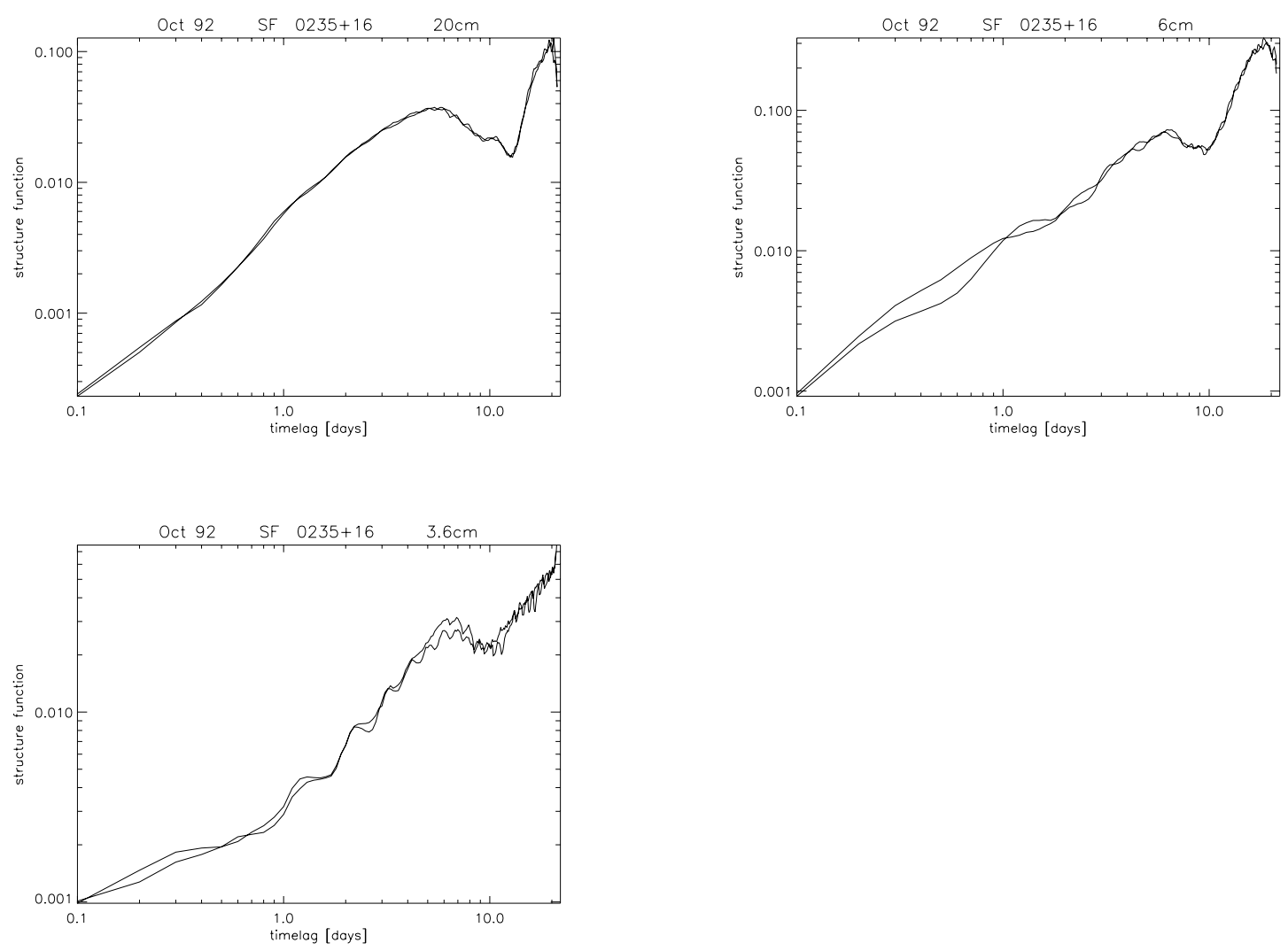

Fig. 34. Structure functions October 1992; 0235+164
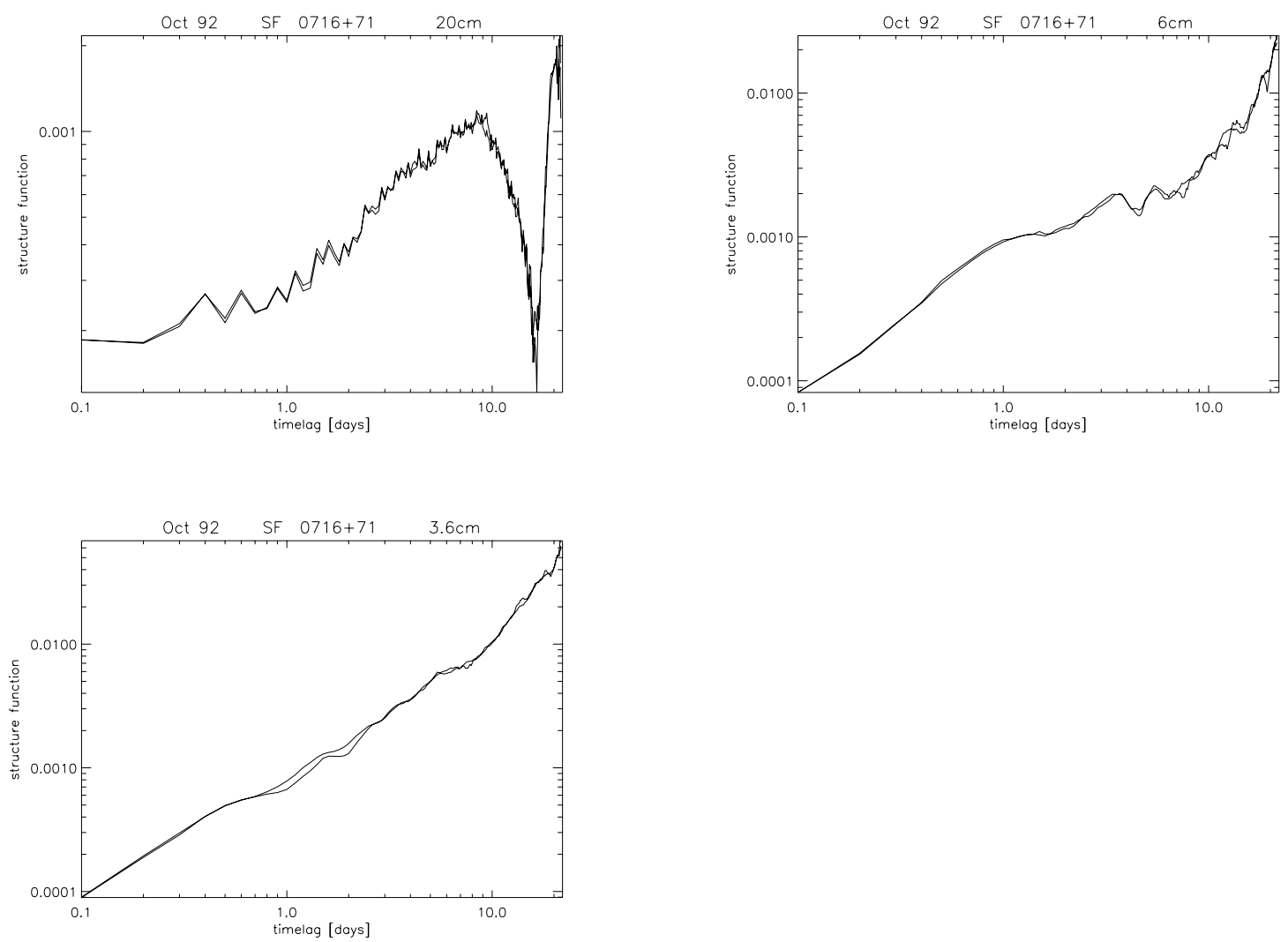

Fig. 35. Structure functions October 1992; 0716+714 
A. Quirrenbach et al.: Intraday variability in compact extragalactic radio sources. I.
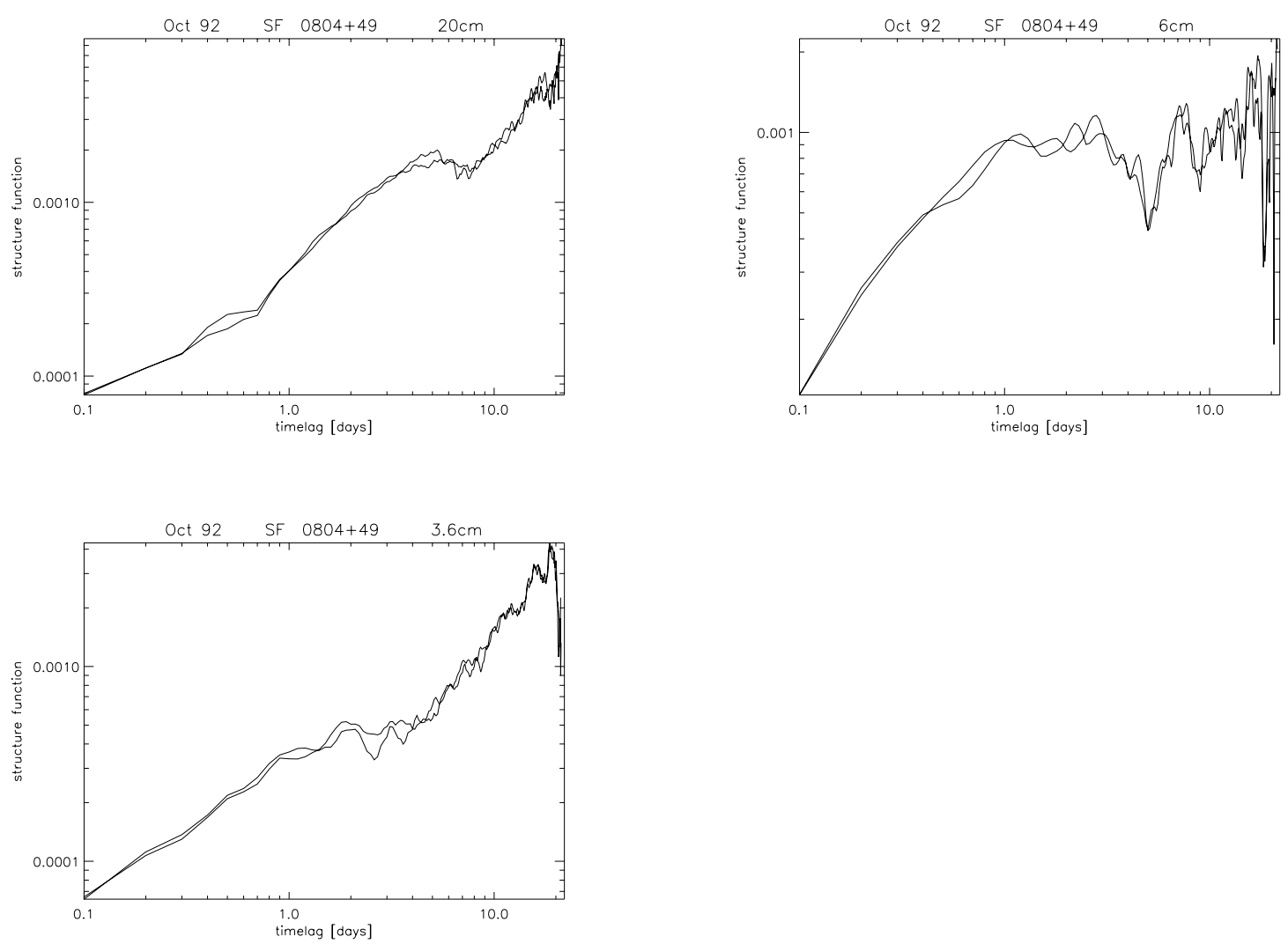

Fig. 36. Structure functions October 1992; 0804+499
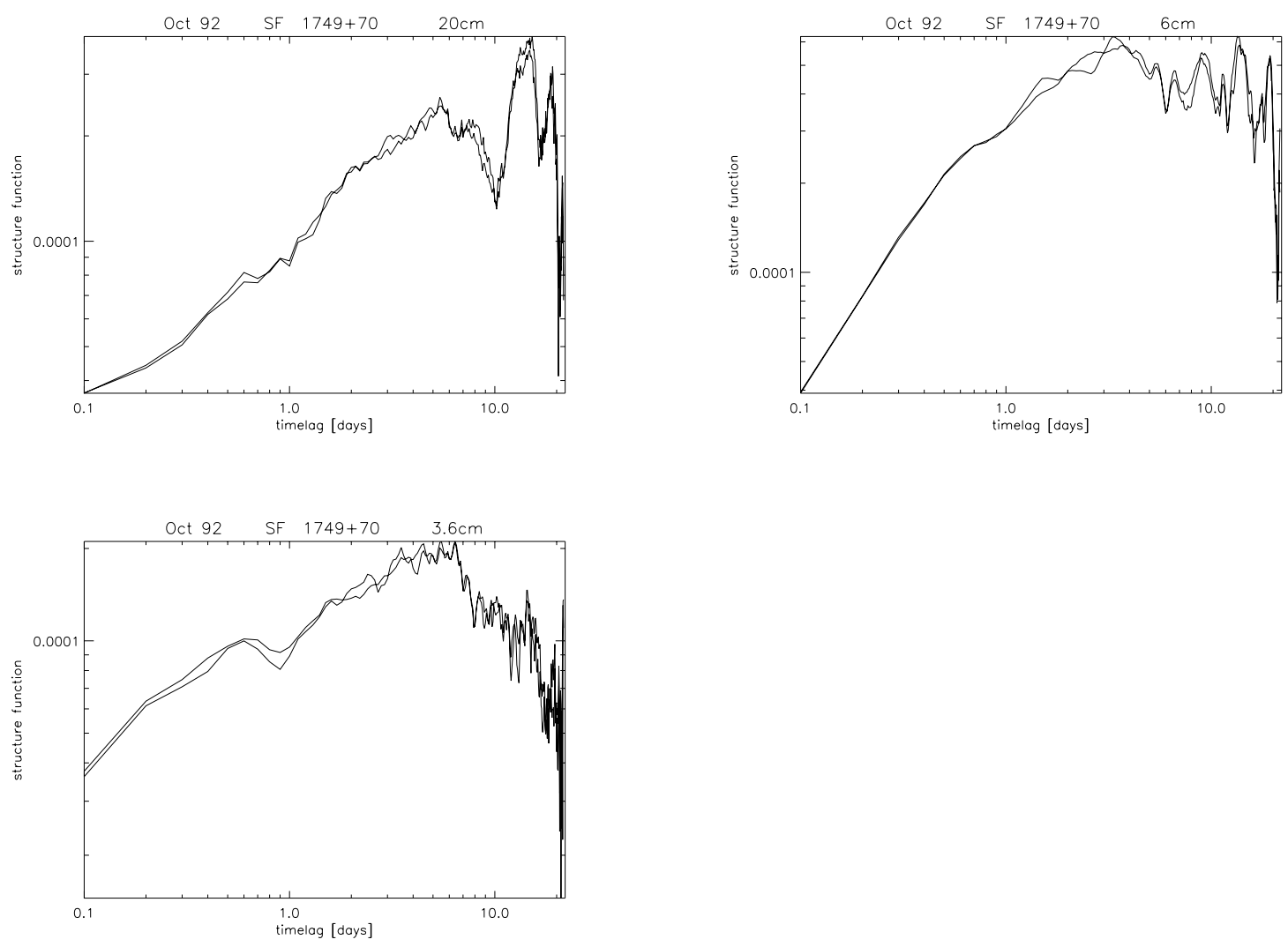

Fig. 37. Structure functions October 1992; 1749+701 
A. Quirrenbach et al.: Intraday variability in compact extragalactic radio sources. I.
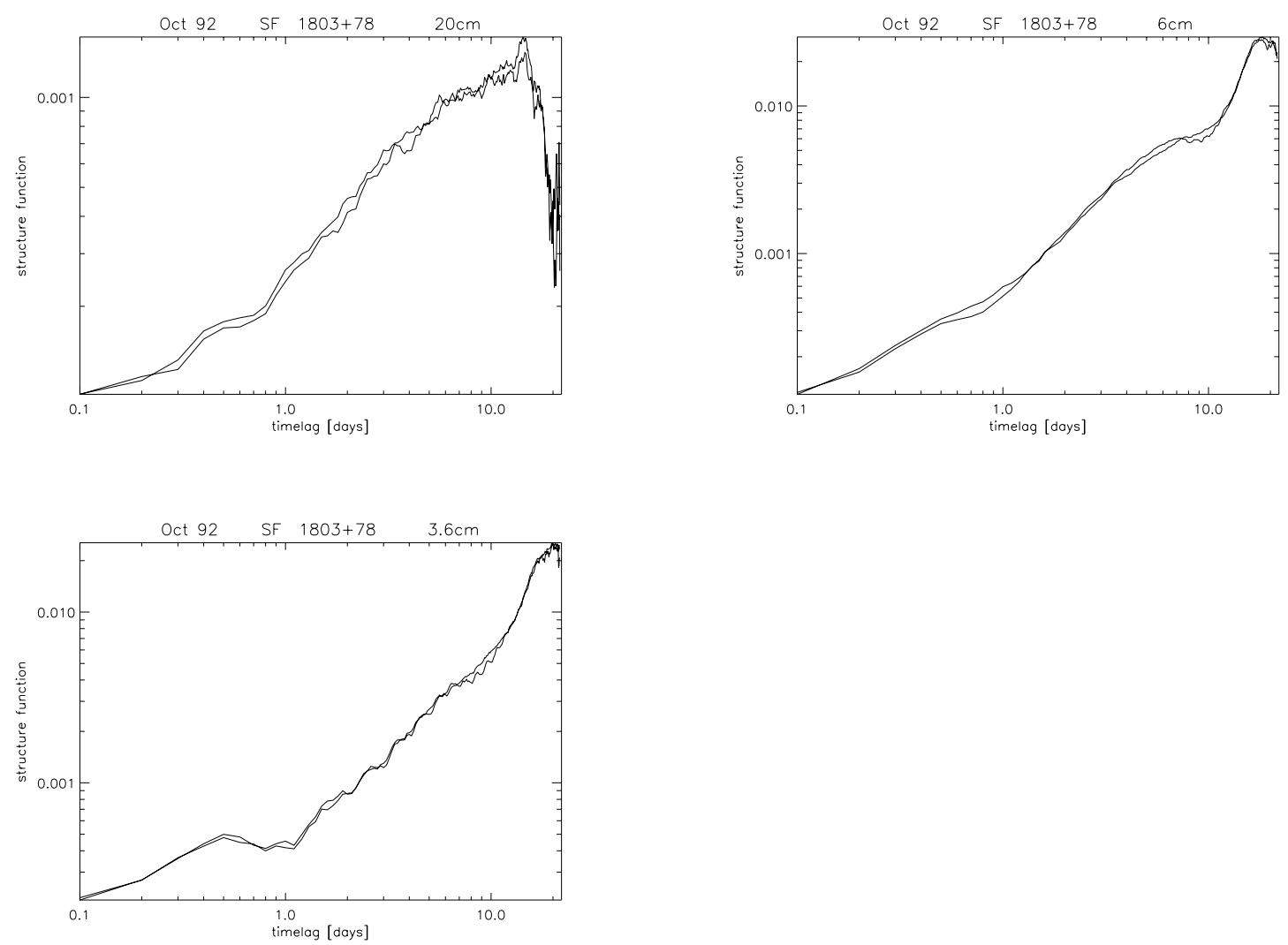

Fig. 38. Structure functions October 1992; 1803+784
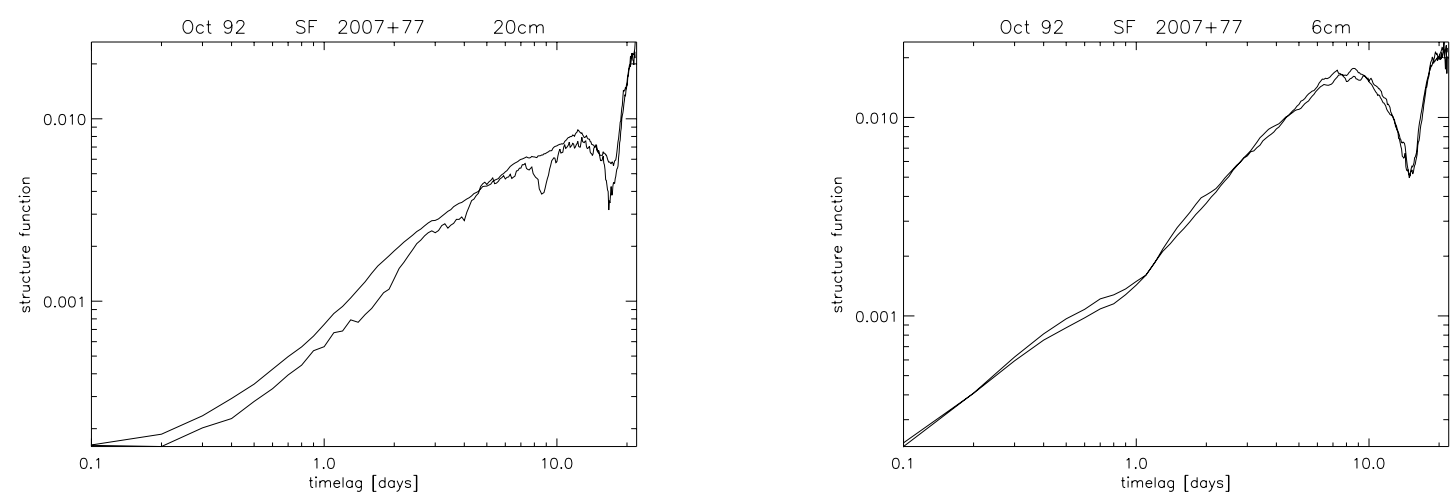

Fig. 39. Structure functions October 1992; 2007+777
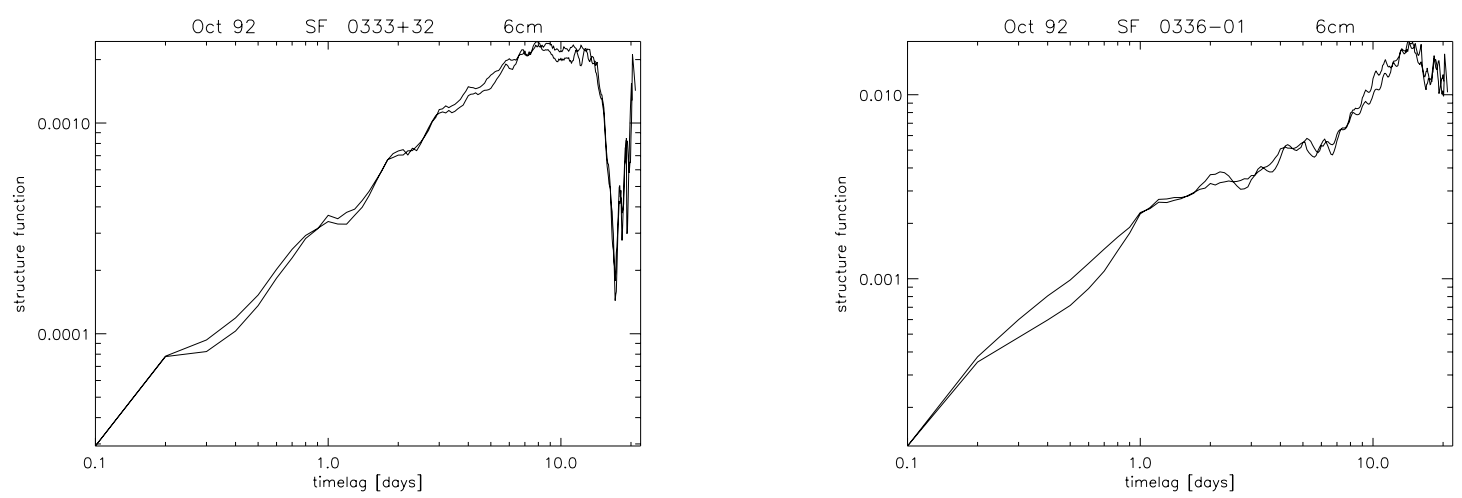

Fig. 40. Structure functions October 1992; $\lambda=6 \mathrm{~cm}, 0333+321,0336-019$ 